CUBO A Mathematical Journal Vol.13, № 03, (153-184). October 2011

\title{
Sum and Difference Compositions in Discrete Fractional Calculus
}

\author{
Michael Holm \\ The University of Nebraska-Lincoln,
}

\begin{abstract}
We introduce fractional sum and difference operators, study their behavior and develop a complete theory governing their compositions. This theory is then applied to solve a general, fractional initial value problem.
\end{abstract}

\section{RESUMEN}

Introducimos operadores de suma y diferencia fraccionaria, estudiamos su comportamiento y desarrollamos una teora completa que rige sus composiciones. Aplicamos esta teoría para resolver un problema fraccionado de valor inicial.

Keywords: Discrete Fractional Calculus, Fractional Sum, Fractional Difference, Composition Rule, Fractional Initial Value Problem.

Mathematics Subject Classification: 34

\section{Introduction}

Gottfried Leibniz and Guilliaume L'Hôpital are believed to have first sparked curiosity into the idea of fractional calculus during a 1695 overseas correspondence on the possible meaning for

\footnotetext{
${ }^{1}$ A special thank you to Dr. Allan Peterson for his input throughout my work on this paper.
} 
a one-half derivative. By the late $19^{\text {th }}$-century, combined efforts by several mathematicians-most notably Liouville, Grünwald, Letnikov and Riemann-led to a fairly solid understanding of fractional calculus in the continuous setting. Since then, fractional calculus has been used as a tool to study and model a variety of applied problems. Indeed, Podlubney [9] outlines fractional calculus applications in the fields of viscoelasticity, capacitor theory, electrical circuits, electroanalytical chemistry, neurology, diffusion, control theory and statistics.

Significantly less is known, however, about discrete fractional calculus. To the author's knowledge, significant work to develop this area did not appear until the mid-1950's, with the majority of interest shown within the past thirty years. Diaz and Osler [4] published a 1974 paper introducing a fractional difference defined as an infinite series, a generalization of the binomial formula for the $\mathrm{N}^{\text {th }}$-order difference $\Delta^{\mathrm{N}}$ f. Although this definition agrees with the one presented in this paper for whole-order differences, it differs elsewhere.

The fractional difference given in this paper is based on the one first given by Gray and Zhang [5] in 1988. They developed a special case for one composition rule given in this paper as well as versions of a power rule and Leibniz' formula. However, Gray and Zhang worked exclusively with the nabla operator, and so their results still differ from the few corresponding results found in this paper.

A recent interest in discrete fractional calculus has been shown by Atici and Eloe, who discussed in 1] properties of the generalized falling function, a corresponding power rule for fractional delta-operators and the commutivity of fractional sums. One year later, they presented in 2 more rules for composing fractional sums and differences, but they left many important cases unresolved or untouched. In addition, [1] and 2] pay little attention to function domains or to lower limits of summation and differentiation, two details vital for a correct and careful treatment of the power rule and the sum and difference composition rules - their neglect leads to domain confusion and, worse, to false or ambiguous claims.

The goal of this paper is to develop and present a complete and precise theory for composing fractional sums and differences. Careful attention is given to detail as several side matters are addressed along the way, including correcting and broadening the power rule stated incorrectly in [1, 2] and [3. We conclude this paper in Section 4 by using the tools developed in Sections 1-3 to solve a general fractional initial value problem.

\subsection{Motivation}

We consider throughout this paper real-valued functions defined on a shift of the natural numbers:

$$
f: \mathbb{N}_{a} \rightarrow \mathbb{R} \text {, where } \mathbb{N}_{a}:=\mathbb{N}_{0}+\{a\}=\{a, a+1, a+2, \ldots\} \quad(a \in \mathbb{R} \text { fixed })
$$


Just as

$$
y(t)=\int_{a}^{t} \frac{(t-s)^{n-1}}{(n-1) !} f(s) d s, t \in[a, \infty)
$$

is the unique solution to the initial-value problem

$$
\left\{\begin{array}{l}
y^{(n)}(t)=f(t), \quad t \in[a, \infty) \\
y^{(i)}(a)=0, \quad i=0,1, \ldots, n-1
\end{array},\right.
$$

so the discrete function

$$
y(t)=\sum_{s=a}^{t-n} \frac{(t-s-1) \frac{n-1}{(n-1) !}}{(n)}(s), t \in \mathbb{N}_{a}
$$

is the unique solution to the initial-value problem

$$
\left\{\begin{array}{l}
\Delta^{n} y(t)=f(t), \quad t \in \mathbb{N}_{a} \\
\Delta^{i} y(a)=0, \quad i=0,1, \ldots, n-1
\end{array} .\right.
$$

In this latter case, it is easy to see that the solution $y$ must satisfy

$$
y(a)=y(a+1)=\cdots=y(a+n-1)=0
$$

and that $y$ is found by simplifying $n$ iterated sums of $f$, each taken from $\tau=a$ to $\tau=t$ (See [6]). We denote this solution $y$ with the symbol $\Delta_{a}^{-n} f$, and we call $y=\Delta_{a}^{-n} f$ the $n^{\text {th }}$-order sum of $f$.

\subsection{Definitions}

The above discussion motivates the following definition for an arbitrary, real-order sum.

Definition 1.1. Let $f: \mathbb{N}_{a} \rightarrow \mathbb{R}$ and $v>0$ be given. Then the $v^{\text {th }}$-order fractional sum of $f$ ("the $v^{\text {th }}$-sum of $f$ ") is given by

$$
\left(\Delta_{a}^{-v} f\right)(t):=\frac{1}{\Gamma(v)} \sum_{s=a}^{t-v}(t-\sigma(s)) \frac{v-1}{f} f(s), \quad \text { for } t \in \mathbb{N}_{a+v} .
$$

Also, we define the trivial sum $\Delta_{\mathrm{a}}^{-0} \mathrm{f}(\mathrm{t}):=\mathrm{f}(\mathrm{t})$, for $\mathrm{t} \in \mathbb{N}_{\mathrm{a}}$.

Remark 1.1. - The name fractional sum is a misnomer, strictly speaking. Early mathematicians working in the area had in mind rational order operators, but both in the general theory and in this paper, we allow sums of arbitrary real order-the symbols $\Delta_{\mathrm{a}}^{-5} \mathrm{f}, \Delta_{\mathrm{a}}^{-\frac{7}{3}} \mathrm{f}, \Delta_{\mathrm{a}}^{-\sqrt{2}} \mathrm{f}$ and $\Delta_{\mathrm{a}}^{-\pi} \mathrm{f}$ all represent legitimate fractional sums.

- For ease of notation, we will throughout this paper use the symbol $\Delta_{\mathrm{a}}^{-v} \mathrm{f}(\mathrm{t})$ in place of $\left(\Delta_{\mathrm{a}}^{-v} \mathrm{f}\right)(\mathrm{t})$, where the $\mathrm{t}$ in $\Delta_{\mathrm{a}}^{-v} \mathrm{f}(\mathrm{t})$ represents an input for the function $\Delta_{\mathrm{a}}^{-v} \mathrm{f}$, and not for the function $\mathrm{f}$. 
- The fractional sum $\Delta_{\mathrm{a}}^{-v} \mathrm{f}(\mathrm{t})$ is a definite integral and depends on its lower limit of summation a. In fact, it makes sense to write $\Delta_{\mathrm{a}}^{-v} \mathrm{f}$ only if we know a priori that $\mathrm{f}$ is defined on $\mathbb{N}_{\mathrm{a}}$. The lower limit of summation, therefore, provides us with an important tool for keeping correct track of function domains throughout our work-omitting the subscript leads to domain confusion and general ambiguity.

- The $\sigma$-function is used in (1.1) because of its tie to the more general theory of time scales, where for a discrete time scale such as $\mathbb{N}_{\mathrm{a}}, \sigma(\mathrm{s})$ denotes the next point in the time scale after s. In this case, $\sigma(\mathrm{s})=\mathrm{s}+1$, for all $\mathrm{s} \in \mathbb{N}_{\mathrm{a}}$.

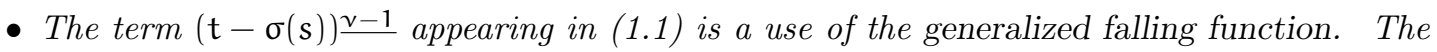
generalized falling function is given by

$$
\mathrm{t}^{\underline{v}}:=\frac{\Gamma(\mathrm{t}+1)}{\Gamma(\mathrm{t}+1-v)}
$$

for any $\mathrm{t}, \boldsymbol{v} \in \mathbb{R}$ for which the right-hand side is well-defined. Hence,

$$
(\mathrm{t}-\sigma(\mathrm{s})) \frac{v-1}{\Gamma(\mathrm{t}-\mathrm{s})}=\frac{\mathrm{t}-\mathrm{s}-\mathrm{v}+1)}{\Gamma}
$$

We will also use the identities

$$
v^{\underline{v}}=\Gamma(v+1), \Delta \mathrm{t}^{\underline{v}}=v \mathrm{t}^{\underline{v-1}} \text { and } \mathrm{t} \underline{v+1}=(\mathrm{t}-v) \mathrm{t}^{\underline{v}},
$$

which hold whenever both sides are well-defined (See Theorem 3.1 for a proof of the second identity).

With a definition for the $v^{\text {th }}$-fractional sum in hand, we give the following traditional definition for the $v^{\text {th }}$-fractional difference 7 .

Definition 1.2. Let $\mathrm{f}: \mathbb{N}_{\mathrm{a}} \rightarrow \mathbb{R}$ and $v \geq 0$ be given, and let $\mathrm{N} \in \mathbb{N}$ be chosen such that $\mathrm{N}-1<v \leq \mathrm{N}$. Then the $v^{\mathrm{th}}$-order fractional difference of $\mathrm{f}$ ("the $v^{\mathrm{th}}$-difference of $\mathrm{f}$ ") is given by

$$
\left(\Delta_{a}^{v} f\right)(t)=\Delta_{a}^{v} f(t):=\Delta^{N} \Delta_{a}^{-(N-v)} f(t), \text { for } t \in \mathbb{N}_{a+N-v} .
$$

Remark 1.2. - In this traditional definition, fractional-order differences are defined as the next higher whole-order difference acting on a small-order sum. Later (Theorem 2.1), we will derive an equivalent form for $\Delta_{\mathrm{a}}^{v} \mathrm{f}$ whose use is essential in many applications.

- Definition 1.2 agrees with the definition for standard whole-order differences: For any $v=$ $\mathrm{N} \in \mathbb{N}_{0}$

$$
\Delta_{a}^{v} f(t)=\Delta^{N} \Delta_{a}^{-(N-v)} f(t)=\Delta^{N} \Delta_{a}^{-0} f(t)=\Delta^{N} f(t), \text { for } t \in \mathbb{N}_{a} .
$$

- When using (1.2), we often wish to apply the following well-known binomial formula for the whole-order difference $\Delta^{\mathrm{N}}$ :

$$
\Delta^{N} f(t)=\sum_{i=0}^{N}(-1)^{i}\left(\begin{array}{c}
N \\
i
\end{array}\right) f(t+N-i) .
$$


- It is important to note that, whereas whole-order differences do not depend on any starting point or lower limit a, fractional differences do. To demonstrate, we know that the second difference of a function $\mathrm{f}: \mathbb{N}_{\mathrm{a}} \rightarrow \mathbb{R}$ at any point $\mathrm{t} \in \mathbb{N}_{\mathrm{a}}$ is given by

$$
\Delta^{2} f(t)=f(t+2)-2 f(t+1)+f(t)
$$

an expression that in no way depends on a, but only on the values $f$ takes on the set $\{t, t+1, t+2\}$. However, the fractional difference

$$
\Delta_{a}^{1.5} f(t)=\Delta^{2} \Delta_{a}^{-0.5} f(t)=\Delta_{a}^{-0.5} f(t+2)-2 \Delta_{a}^{-0.5} f(t+1)+\Delta_{a}^{-0.5} f(t)
$$

does depend on the lower limit of summation a. This dependence frustrates our traditional notion of a difference. However, the dependence of $\Delta_{\mathrm{a}}^{\mathrm{v}} \mathrm{f}$ on a does vanish as $\mathrm{v} \rightarrow \mathrm{N}^{-}$(see Theorem 2.2). For this reason, we write $\Delta^{\mathrm{N}_{f}}$ for whole-order differences and $\Delta_{\mathrm{a}}^{\mathrm{v}}$ for general fractional differences.

- In the above setting, it is important to think of $v>0$ as being situated between two natural numbers. For such a $v$ with $\mathrm{N}-1<v \leq \mathrm{N}\left(\mathrm{N} \in \mathbb{N}_{0}\right)$, we call the fractional difference equation $\Delta_{\mathrm{a}+\boldsymbol{v}-\mathrm{N}}^{\mathrm{y}}(\mathrm{t})=\mathrm{f}(\mathrm{t})$ a $v^{\mathrm{th}}$-order equation, and we identify it with and compare it to the whole-order difference equation $\Delta^{\mathrm{N}} \mathrm{y}(\mathrm{t})=\mathrm{f}(\mathrm{t})$.

\subsection{Domains}

When working with fractional sums and differences, it is crucial to understand their domains. We first look at domains for sums and then at domains for differences.

Consider the first-order definite sum of a function $f$ at a point $t \in \mathbb{N}_{a}$, for which we sum up the values of $f(\tau)$ from $\tau=a$ to $\tau=t-1$. As we know, this definite sum $\Delta_{a}^{-1} f(t)$ represents the 'area' under the graph of $f$ from a to $t$, where the height of the function on the interval $[t, t+1]$ is given by the value $f(t)$. In light of this, one may choose to consider the value $\Delta_{a}^{-1} f(a)$, thinking of this as the trivial area under $f$ from $t=a$ to $t=a$, which is zero. Using Definition 1.1 for $\Delta_{\mathrm{a}}^{-1} \mathrm{f}(\mathrm{a})$, we find that

$$
\Delta_{a}^{-1} f(a)=\left.\frac{1}{\Gamma(1)} \sum_{s=a}^{t-1}(t-\sigma(s)) \frac{1-1}{-a} f(s)\right|_{t=a}=\sum_{s=a}^{a-1} f(s) .
$$

Therefore, if we insist on considering $\Delta_{a}^{-1} f(a)$ as a legitimate value, then we must hold the convention that $\sum_{s=a}^{a-1} f(s)=0$.

Likewise, if we recognize the $N$ values $\Delta_{a}^{-N} f(a), \Delta_{a}^{-N} f(a+1), \cdots, \Delta_{a}^{-N} f(a+N-1)$ as having legitimate mathematical meaning, we must have

$$
\Delta_{\mathrm{a}}^{-\mathrm{N}} \mathrm{f}(\mathrm{a})=\Delta_{\mathrm{a}}^{-\mathrm{N}} \mathrm{f}(\mathrm{a}+1)=\cdots=\Delta_{\mathrm{a}}^{-\mathrm{N}} \mathrm{f}(\mathrm{a}+\mathrm{N}-1)=0,
$$


which leads us to adopt the convention that

$$
\frac{1}{\Gamma(N)} \sum_{s=a}^{a+i-N}(a+i-\sigma(s)) \frac{N-1}{f} f(s)=0, \text { for } i \in\{0,1, \ldots, N-1\} .
$$

This idea extends further to fractional sums as follows: For any $v>0$ with $N-1<v \leq N$,

$$
\Delta_{a}^{-v} f(a+v-N)=\Delta_{a}^{-v} f(a+v-N+1)=\cdots=\Delta_{a}^{-v} f(a+v-1)=0 .
$$

In light of this, it is convenient and sensible to ignore these zeros and to define

$$
\mathcal{D}\left\{\Delta_{\mathrm{a}}^{-v} \mathrm{f}\right\}:=\mathbb{N}_{\mathrm{a}+v}
$$

as given in Definition 1.1. It can be shown that the first nontrivial value $\Delta_{\mathrm{a}}^{-v} f$ takes on is the value

$$
\Delta_{a}^{-v} f(a+v)=f(a) .
$$

However, we will at certain times recall and use the fact that the above discussed zeros exist before this point in the domain.

We next use fractional sum domains to determine fractional difference domains. Whereas whole-order differences are domain preserving operators (i.e. $\mathcal{D}\left(\Delta^{N_{f}}\right)=\mathcal{D}(f)$, for all $N \in \mathbb{N}_{0}$ ), we find that a fractional difference operator shifts the domain of its argument. Using Definition 1.2 we find that for $f: \mathbb{N}_{a} \rightarrow \mathbb{R}$ and $v>0$ with $N-1<v \leq N$,

$$
\mathcal{D}\left\{\Delta_{\mathrm{a}}^{v} \mathrm{f}\right\}=\mathcal{D}\left\{\Delta^{\mathrm{N}} \Delta_{\mathrm{a}}^{-(\mathrm{N}-v)} \mathrm{f}\right\}=\mathcal{D}\left\{\Delta_{\mathrm{a}}^{-(\mathrm{N}-v)} \mathrm{f}\right\}=\mathbb{N}_{\mathrm{a}+\mathrm{N}-\mathrm{v}}
$$

Note that whereas the domain shift for a fractional sum is a large shift by $v$ to the right, the domain shift for a fractional difference is a relatively small shift by $\mathrm{N}-v$ to the right.

We next focus on the domains of two-operator sum and difference compositions. Consider, for example, the composition

$$
\left.\Delta_{a+\mu}^{-v} \Delta_{a}^{-\mu} f(t)=\frac{1}{\Gamma(\nu)} \sum_{s=a+\mu}^{t-v}(t-\sigma(s)) \frac{v-1}{\Gamma(\mu)} \sum_{r=a}^{s-\mu}(s-\sigma(r)) \frac{\mu-1}{f} f(r)\right) .
$$

Notice that the lower limit of the outer operator $\Delta_{\mathrm{a}+\mu}^{-v}$ must match the domain of the inner function $\Delta_{\mathrm{a}}^{-\mu} \mathrm{f}(\mathrm{t})$, which is $\mathbb{N}_{\mathrm{a}+\mu}$. Hence, the domain of the entire composition is $\mathbb{N}_{\mathrm{a}+\mu+v}$. The summary below shows the domains of all four possible sum and difference compositions.

Summary 1.1. $\quad$ Let $\mathrm{f}: \mathbb{N}_{\mathrm{a}} \rightarrow \mathbb{R}$ and $\nu, \mu>0$ be given, with $\mathrm{N}-1<\nu \leq \mathrm{N}$ and $\mathrm{M}-1<\mu \leq$ M. Then

- $\mathcal{D}\left\{\Delta_{\mathrm{a}}^{-v} \mathrm{f}\right\}=\mathbb{N}_{\mathrm{a}+\boldsymbol{v}}$

- $\mathcal{D}\left\{\Delta_{\mathrm{a}+\mu}^{-v} \Delta_{\mathrm{a}}^{-\mu} \mathrm{f}\right\}=\mathbb{N}_{\mathrm{a}+\mu+v}$

- $\mathcal{D}\left\{\Delta_{a+M-\mu}^{-v} \Delta_{a}^{\mu} f\right\}=\mathbb{N}_{a+M-\mu+v}$
- $\mathcal{D}\left\{\Delta_{\mathrm{a}}^{v} \mathrm{f}\right\}=\mathbb{N}_{\mathrm{a}+\mathrm{N}-v}$

- $\mathcal{D}\left\{\Delta_{\mathrm{a}+\mu}^{v} \Delta_{\mathrm{a}}^{-\mu} f\right\}=\mathbb{N}_{\mathrm{a}+\mu+\mathrm{N}-v}$

- $\mathcal{D}\left\{\Delta_{\mathrm{a}+\mathrm{M}-\mu}^{\nu} \Delta_{\mathrm{a}}^{\mu} f\right\}=\mathbb{N}_{\mathrm{a}+\mathrm{M}-\mu+\mathrm{N}-v}$ 


\section{Unifying the Fractional Sum and Difference}

We show here that fractional sums and differences can be unified by a common definition, for which an appropriate version of Leibniz' Rule is useful.

Let $g: \mathbb{N}_{a+v} \times \mathbb{N}_{a} \rightarrow \mathbb{R}$ be given. Then

$$
\Delta\left(\sum_{s=\mathrm{a}}^{\mathrm{t}-\boldsymbol{v}} \mathrm{g}(\mathrm{t}, \mathrm{s})\right)=\sum_{s=\mathrm{a}}^{\mathrm{t}-\boldsymbol{v}} \Delta_{\mathrm{t}} \mathrm{g}(\mathrm{t}, \mathrm{s})+\mathrm{g}(\mathrm{t}+1, \mathrm{t}+1-v), \text { for } \mathrm{t} \in \mathbb{N}_{\mathrm{a}+\boldsymbol{v}} .
$$

Leibniz' Rule (2.1) is used in proving the following theorem, which gives a different but equivalent way of defining a fractional difference, one which mirrors the definition for the fractional sum. Moreover, Theorem 2.1 will allow us to substantially extend results from previous papersmost notably the power and composition rules.

Theorem 2.1. Let $\mathrm{f}: \mathbb{N}_{\mathrm{a}} \rightarrow \mathbb{R}$ and $v>0$ be given, with $\mathrm{N}-1<v \leq \mathrm{N}$. Then the following two definitions for the fractional difference $\Delta_{\mathrm{a}}^{v} \mathrm{f}: \mathbb{N}_{\mathbf{a}+\mathrm{N}-v} \rightarrow \mathbb{R}$ are equivalent:

$$
\begin{gathered}
\Delta_{a}^{v} f(t):=\Delta^{N} \Delta_{a}^{-(N-v)} f(t), \\
\Delta_{a}^{v} f(t):=\left\{\begin{array}{lc}
\frac{1}{\Gamma(-v)} \sum_{s=a}^{t+v}(t-\sigma(s)) \frac{-v-1}{\frac{}{N}} f(s), & N-1<v<N \\
\Delta^{N} f(t), & v=N
\end{array}\right.
\end{gathered}
$$

Proof. Let $f$ and $v$ be given as in the statement of the theorem. We assume that (2.2) is the correct definition for the fractional difference and show that (2.3) is equivalent, for $t \in \mathbb{N}_{a+N-v}$.

If $v=\mathrm{N}$, then definitions (2.2) and (2.3) are clearly equivalent, since

$$
\Delta_{a}^{v} f(t)=\Delta^{N} \Delta_{a}^{-(N-v)} f(t)=\Delta^{N} \Delta_{a}^{-0} f(t)=\Delta^{N} f(t) .
$$

If $\mathrm{N}-1<v<\mathrm{N}$, then direct application of (2.2) yields

$$
\begin{aligned}
& \Delta_{a}^{v} f(t) \\
& =\Delta^{N} \Delta_{a}^{-(N-v)} f(t) \\
& =\Delta^{N}\left[\frac{1}{\Gamma(N-v)} \sum_{s=a}^{t-(N-v)}(t-\sigma(s)) \frac{N-v-1}{f} f(s)\right] \\
& \left.=\frac{\Delta^{N-1}}{\Gamma(N-v)} \cdot \Delta\left[\sum_{s=a}^{t-(N-v)}(t-\sigma(s)) \frac{N-v-1}{f} f(s)\right] \text { (apply (2.1) }\right), \\
& =\frac{\Delta^{N-1}}{\Gamma(N-v)}\left[\sum_{s=a}^{t-(N-v)}\left((N-v-1)(t-\sigma(s)) \frac{N-v-2}{2} f(s)\right)\right.
\end{aligned}
$$




$$
\begin{aligned}
& \left.+(t+1-\sigma(t+1-(N-v))) \frac{N-v-1}{f} f(t+1-(N-v))\right] \\
= & \Delta^{N-1}\left[\sum_{s=a}^{t-(N-v)} \frac{(t-\sigma(s)) \frac{N-v-2}{\Gamma(N-v-1)}}{\Gamma(s)+f(t+1-(N-v))]}\right. \\
= & \Delta^{N-1}\left[\frac{1}{\Gamma(N-v-1)} \sum_{s=a}^{t+1-(N-v)}(t-\sigma(s)) \frac{N-v-2}{f} f(s)\right] \\
= & \Delta^{N-1}\left[\frac{1}{\Gamma(N-v-1)} \sum_{s=a}^{t-(N-v-1)}(t-\sigma(s)) \frac{N-v-2}{f} f(s)\right] .
\end{aligned}
$$

Repeating these steps $\mathrm{N}-2$ more times, we find that

$$
\begin{aligned}
& \Delta_{a}^{v} f(t)=\Delta^{N-1}\left[\frac{1}{\Gamma(N-v-1)} \sum_{s=a}^{t-(N-v-1)}(t-\sigma(s)) \frac{N-v-2}{N} f(s)\right] \\
& =\Delta^{\mathrm{N}-2}\left[\frac{1}{\Gamma(\mathrm{N}-v-2)} \sum_{s=\mathrm{a}}^{\mathrm{t}-(\mathrm{N}-\mathrm{v}-2)}(\mathrm{t}-\sigma(\mathrm{s})) \frac{\mathrm{N}-\boldsymbol{v}-3}{\mathrm{f}} \mathrm{f}(\mathrm{s})\right] \\
& =\Delta^{N-N}\left[\frac{1}{\Gamma(N-v-N)} \sum_{s=a}^{t-(N-v-N)}(t-\sigma(s)) \frac{N-v-(N+1)}{N} f(s)\right] \\
& =\frac{1}{\Gamma(-v)} \sum_{s=\mathrm{a}}^{\mathrm{t}+\boldsymbol{v}}(\mathrm{t}-\sigma(s)) \frac{-v-1}{-} f(s) .
\end{aligned}
$$

Note that since $\mathrm{N}-1<v<\mathrm{N}$ in the above work, the term $\frac{1}{\Gamma(\mathrm{N}-v-\mathrm{k})}$ exists for each $k=1,2, \ldots, N$. Furthermore, for each point $t \in \mathbb{N}_{a+N-v}$ (say $t=a+N-v+m$, for some $\left.m \in \mathbb{N}_{0}\right)$,

$$
(\mathrm{t}-\sigma(\mathrm{s})) \frac{\mathrm{N}-\mathrm{v}-\mathrm{k}-1}{\Gamma(\mathrm{t}-\mathrm{s}-\mathrm{N}+\mathrm{s}+\mathrm{k}+1)}=\frac{\Gamma(\mathrm{a}+\mathrm{N}-\mathrm{v}+\mathrm{m}-\mathrm{s})}{\Gamma(\mathrm{a}+\mathrm{m}+\mathrm{k}+1-\mathrm{s})}
$$

exists and is well-defined for each $k \in\{1,2, \ldots, N\}$ and

$$
s \in\{a, a+1, \ldots, t-(N-v-k)\}=\{a, a+1, \ldots, a+m+k\} .
$$

Note, finally, that although definition (2.3) appears to be valid for all $t \in \mathbb{N}_{a-v}$, it only defines the $v^{\text {th }}$-fractional difference on $\mathbb{N}_{\mathrm{a}+\mathrm{N}-\boldsymbol{v} \text {. }}$

In definition (2.3), one may wonder about the continuity of $\Delta_{\mathrm{a}}^{v} \mathrm{f}$ with respect to $v$. We certainly desire for every function $f: \mathbb{N}_{a} \rightarrow \mathbb{R}$, for example, that $\Delta_{a}^{1.99} f$ be very close to $\Delta^{2} f$. However, the term $\frac{1}{\Gamma(-v)}$ in (2.3) blows up as $v \rightarrow N^{-}$! The following theorem addresses this matter. 
Theorem 2.2. Let $\mathrm{f}: \mathbb{N}_{\mathrm{a}} \rightarrow \mathbb{R}$ be given. Then the fractional difference $\Delta_{\mathrm{a}}^{v_{\mathrm{f}}}$ is continuous with respect to $v \geq 0$. More specifically, for each $v>0$, let $t_{v, m}:=a+\lceil\nu\rceil-v+m$ be a fixed but arbitrary point in $\mathcal{D}\left\{\Delta_{a}^{v} \mathrm{f}\right\}$. Then for each $\mathrm{m} \in \mathbb{N}_{0}$,

$$
v \mapsto \Delta_{\mathrm{a}}^{v} \mathrm{f}\left(\mathrm{t}_{\mathrm{v}, \mathrm{m}}\right) \text { is continuous on }[0, \infty) \text {. }
$$

Proof. Let $f: \mathbb{N}_{a} \rightarrow \mathbb{R}$ be given, and fix $N \in \mathbb{N}$ and $m \in \mathbb{N}_{0}$. It is enough to show that

$$
\begin{aligned}
& \Delta_{a}^{v} f(a+N-v+m) \text { is continuous with respect to } v \text { on }(N-1, N) \\
& \Delta_{a}^{v} f(a+N-v+m) \rightarrow \Delta^{N} f(a+m) \text { as } v \rightarrow N^{-} \\
& \Delta_{a}^{v} f(a+N-v+m) \rightarrow \Delta^{N-1} f(a+m+1) \text { as } v \rightarrow(N-1)^{+}
\end{aligned}
$$

Note that for any fixed $v>0$ with $N-1<v<N$,

$$
\begin{aligned}
& \Delta_{a}^{v} f(a+N-v+m) \\
& =\left.\frac{1}{\Gamma(-v)} \sum_{s=a}^{t+v}(t-\sigma(s)) \frac{-v-1}{f} f(s)\right|_{t=a+N-v+m} \\
& =\frac{1}{\Gamma(-v)} \sum_{s=a}^{a+N+m}(a+N-v+m-\sigma(s)) \frac{-v-1}{f} f(s) \\
& =\frac{1}{\Gamma(-v)} \sum_{s=a}^{a+N+m} \frac{\Gamma(a+N-v+m-s)}{\Gamma(a+N+m-s+1)} f(s) \\
& =\sum_{s=a}^{a+N+m} \frac{1}{\Gamma(a+N+m-s+1)} \frac{\Gamma(a+N-v+m-s)}{\Gamma(-v)} f(s) \\
& =\sum_{s=a}^{a+N+m-1}\left(\frac{(a+N-v+m-s-1) \cdots(-v)}{(a+N+m-s) !} f(s)\right)+f(a+N+m) \\
& =\sum_{i=1}^{N+m}\left(\frac{(i-1-v) \cdots(-v+1)(-v)}{i !} f(a+N+m-i)\right)+f(a+N+m) .
\end{aligned}
$$

The above line demonstrates (2.4), the continuity of $v \rightarrow \Delta_{a}^{v} f(a+N-v+m)$ on $(N-1, N)$. Next, we take $v \rightarrow \mathrm{N}^{-}$to show (2.5):

$$
\begin{aligned}
& \lim _{v \rightarrow N^{-}} \Delta_{a}^{v} f(a+N-v+m) \\
& =\lim _{v \rightarrow N^{-}}\left[\sum_{i=1}^{N+m}\left(\frac{(i-1-v) \cdots(-v)}{i !} f(a+N+m-i)\right)+f(a+N+m)\right] \\
& =\sum_{i=1}^{N+m}\left(\frac{(i-1-N) \cdots(-N)}{i !} f(a+N+m-i)\right)+f(a+N+m) \\
& =\sum_{i=1}^{N}\left(\frac{(i-1-N) \cdots(-N)}{i !} f(a+N+m-i)\right)+f(a+N+m)
\end{aligned}
$$


since the argument is zero for $i=N+1, \ldots, N+m$,

$$
\begin{aligned}
& =\sum_{i=1}^{N}\left((-1)^{i} \frac{(N) \cdots(N-i+1)}{i !} f(a+N+m-i)\right)+f(a+N+m) \\
& =\sum_{i=1}^{N}\left((-1)^{i}\left(\begin{array}{c}
N \\
i
\end{array}\right) f(a+N+m-i)\right)+f(a+N+m) \\
& =\sum_{i=0}^{N}(-1)^{i}\left(\begin{array}{c}
N \\
i
\end{array}\right) f(a+N+m-i) \\
& =\sum_{i=0}^{N}(-1)^{i}\left(\begin{array}{c}
N \\
i
\end{array}\right) f((a+m)+N-i) \\
& =\Delta^{N} f(a+m) .
\end{aligned}
$$

Finally, we take $v \rightarrow(\mathrm{N}-1)^{+}$to show (2.6)

$$
\begin{aligned}
& \lim _{v \rightarrow(N-1)^{+}} \Delta_{a}^{v} f(a+N-v+m) \\
= & \lim _{v \rightarrow(N-1)^{+}}\left[\sum_{i=1}^{N+m}\left(\frac{(i-1-v) \cdots(-v)}{i !} f(a+N+m-i)\right)\right. \\
& +f(a+N+m)] \\
= & \sum_{i=1}^{N+m}\left(\frac{(i-N) \cdots(-N+1)}{i !} f(a+N+m-i)\right)+f(a+N+m) \\
= & \sum_{i=1}^{N-1}\left((-1)^{i} \frac{(N-1) \cdots(N-i)}{i !} f(a+N+m-i)\right)+f(a+N+m) \\
= & \sum_{i=1}^{N-1}\left((-1)^{i}\left(\begin{array}{c}
N-1 \\
i
\end{array}\right) f(a+N+m-i)\right)+f(a+N+m) \\
= & \sum_{i=0}^{N-1}\left((-1)^{i}\left(\begin{array}{c}
N-1 \\
i
\end{array}\right) f(a+m+1+(N-1)-i)\right) \\
= & \Delta^{N-1} f(a+m+1) .
\end{aligned}
$$

Remark 2.1. - The above statement (2.7) shows explicitly why a fractional difference's dependence on its lower limit a vanishes as the order of differentiation approaches a whole number.

- Theorem 2.2 implies that for any $f: \mathbb{N}_{a} \rightarrow \mathbb{R}$ and $m \in \mathbb{N}_{0}$, the sequence

$$
\Delta_{a}^{1.9} f(a+m+0.1), \Delta_{a}^{1.99} f(a+m+0.01), \Delta_{a}^{1.999} f(a+m+0.001), \ldots
$$


approaches the value $\Delta^{2} \mathrm{f}(\mathrm{a}+\mathrm{m})$. This notion of "order continuity" adds a beauty to fractional calculus absent from standard whole-order calculus.

Theorem 2.2 shows that definition (2.3) in Theorem 2.1 is a good and reasonable definition for the fractional difference. Hence, we may now unify the fractional sum and difference into a single definition:

Definition 2.1. $\quad$ Let $\mathrm{f}: \mathbb{N}_{\mathrm{a}} \rightarrow \mathbb{R}$ and $\boldsymbol{v}>0$ be given. Then

(i) the $v^{\text {th }}$-fractional sum of $\mathrm{f}$ is given by

$$
\Delta_{a}^{-v} f(t):=\frac{1}{\Gamma(v)} \sum_{s=a}^{t-v}(t-\sigma(s)) \frac{v-1}{f} f(s), t \in \mathbb{N}_{a+v}
$$

(ii) the $v^{\text {th }}$-fractional difference of $\mathrm{f}$ is given by

$$
\Delta_{a}^{v} f(t):=\left\{\begin{array}{ll}
\frac{1}{\Gamma(-v)} \sum_{s=a}^{t+v}(t-\sigma(s)) \frac{-v-1}{-a} f(s), & v \notin \mathbb{N} \\
\Delta^{N} f(t), & v=N \in \mathbb{N}
\end{array}, \quad t \in \mathbb{N}_{a+N-v}\right.
$$

To demonstrate the importance of Definition 2.1, we offer the following theorem generalizing the binomial representation of whole-order differences to fractional sums and differences.

Theorem 2.3. Let $\mathrm{f}: \mathbb{N}_{\mathrm{a}} \rightarrow \mathbb{R}$ and $\boldsymbol{v}>0$ be given, with $\mathrm{N}-1<\boldsymbol{v} \leq \mathrm{N}$.

For each $\mathrm{t} \in \mathbb{N}_{\mathrm{a}+\mathrm{N}-\mathrm{v}}$

$$
\Delta_{a}^{v} f(t)=\sum_{k=0}^{v+t-a}(-1)^{k}\left(\begin{array}{l}
v \\
k
\end{array}\right) f(t+v-k)
$$

For each $\mathrm{t} \in \mathbb{N}_{\mathrm{a}+\boldsymbol{v}}$,

$$
\begin{aligned}
\Delta_{a}^{-v} f(t) & =\sum_{k=0}^{-v+t-a}(-1)^{k}\left(\begin{array}{c}
-v \\
k
\end{array}\right) f(t-v-k) \\
& =\sum_{k=0}^{-v+t-a}\left(\begin{array}{c}
v+k-1 \\
k
\end{array}\right) f(t-v-k) .
\end{aligned}
$$

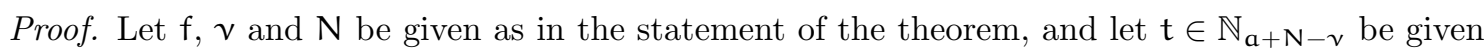


by $t=a+N-v+m$, for some $m \in \mathbb{N}_{0}$. Then

$$
\begin{aligned}
& \Delta_{\mathrm{a}}^{v} \mathrm{f}(\mathrm{t})=\frac{1}{\Gamma(-v)} \sum_{s=\mathrm{a}}^{\mathrm{t}+v}(\mathrm{t}-\sigma(s)) \frac{-v-1}{\mathrm{f}} \mathrm{f}(\mathrm{s}) \\
& =\sum_{s=a}^{t+v} \frac{\Gamma(t-s)}{\Gamma(t-s+v+1) \Gamma(-v)} f(s) \\
& =\sum_{s=a}^{a+N+m} \frac{\Gamma(a+N-v+m-s)}{\Gamma(a+N+m-s+1) \Gamma(-v)} f(s) \\
& =\sum_{s=0}^{N+m} \frac{\Gamma(N+m-s-v)}{\Gamma(N+m-s+1) \Gamma(-v)} f(s+a) \\
& =f(a+N+m)+\sum_{s=0}^{N+m-1} \frac{(N+m-1-s-v) \cdots(-v)}{\Gamma(N+m-s+1)} f(s+a) \\
& =\mathrm{f}(\mathrm{a}+\mathrm{N}+\mathrm{m}) \\
& +\sum_{s=0}^{N+m-1}(-1)^{N+m-s} \frac{(v) \cdots(v-(N+m-s)+1)}{\Gamma(N+m-s+1)} f(s+a) \\
& =\sum_{s=0}^{N+m}(-1)^{N+m-s}\left(\begin{array}{c}
v \\
N+m-s
\end{array}\right) f(s+a) \\
& =\sum_{k=0}^{N+m}(-1)^{k}\left(\begin{array}{l}
v \\
k
\end{array}\right) f(a+N+m-k) \\
& =\sum_{k=0}^{N+m}(-1)^{k}\left(\begin{array}{l}
v \\
k
\end{array}\right) f((a+N-v+m)+v-k) \\
& =\sum_{k=0}^{v+t-a}(-1)^{k}\left(\begin{array}{l}
v \\
k
\end{array}\right) f(t+v-k) \text {, proving (2.8) }
\end{aligned}
$$

Note that when $v=\mathrm{N}$, this reduces to the traditional binomial formula:

$$
\Delta^{N} f(t)=\sum_{k=0}^{N}(-1)^{k}\left(\begin{array}{l}
N \\
k
\end{array}\right) f(t+N-k), \text { for } t \in \mathbb{N}_{a}
$$

A very similar argument proves (2.9). Moreover, when $v=\mathrm{N}$, we interpret

$$
\left(\begin{array}{c}
-\mathrm{N} \\
k
\end{array}\right)=\frac{\Gamma(-\mathrm{N}+1)}{k ! \Gamma(-\mathrm{N}-\mathrm{k}+1)}=\frac{(-\mathrm{N}) \cdots(-\mathrm{N}-\mathrm{k}+1)}{k !}=(-1)^{\mathrm{k}}\left(\begin{array}{c}
\mathrm{N}+\mathrm{k}-1 \\
k
\end{array}\right) \text {. }
$$

In any case, we may write

$$
\left(\begin{array}{c}
-v \\
k
\end{array}\right)=(-1)^{k}\left(\begin{array}{c}
v+k-1 \\
k
\end{array}\right)
$$

whose substitution into (2.9) yields (2.10). Although (2.10) is probably more useful, (2.9) more closely resembles the traditional binomial formula. 


\section{Fractional Sum and Difference Composition Rules}

We turn now to the main focus of this paper. Having set the stage by defining and developing many properties of fractional sums and differences, we have the necessary tools to study the following four compositions:

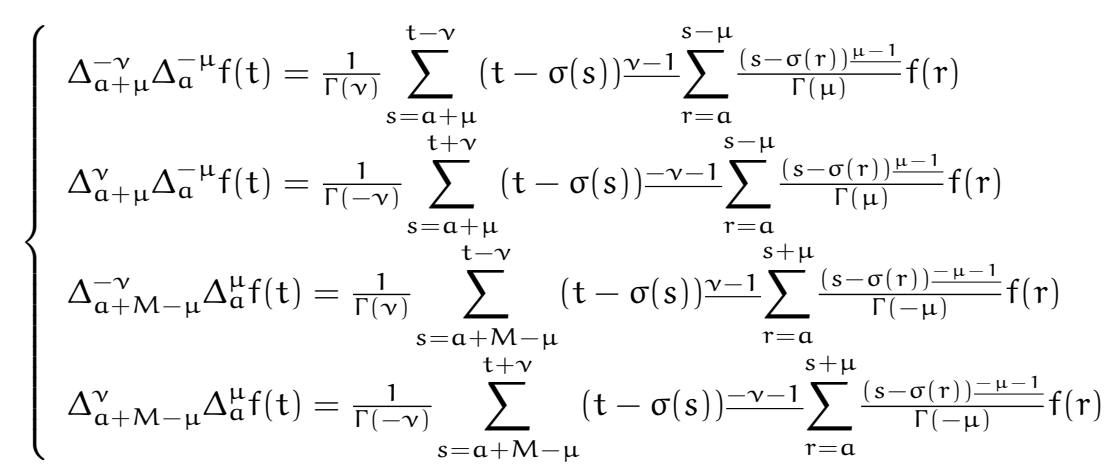

whose domains are as given in Summary 1.1. Definition 2.1 is the tool allowing us to write the above compositions in this more uniform and often more useful way. It will be helpful to keep the above representations and their domains in mind as we develop a rule governing each composition.

To work effectively with these compositions, however, we first need a general and precise power rule for summation and differentiation. Much of the following proof may be found in [1] - however, not having kept track of domains or lower limits, the power rule given in [1] is incorrect as stated. The precise power rule presented in Lemma 3.1 below corrects and extends this previous version.

Lemma 3.1. [Power Rule for Summation and Differentiation]

Let $\mathrm{a} \in \mathbb{R}$ and $\mu>0$ be given. Then,

$$
\Delta(t-a) \underline{\mu}=\mu(t-a) \underline{\mu-1},
$$

for any $\mathrm{t}$ for which both sides are well-defined. Furthermore, for $\boldsymbol{v}>0$ and $\mu \in \mathbb{R} \backslash(-\mathbb{N})$,

$$
\Delta_{\mathrm{a}+\mu}^{-v}(\mathrm{t}-\mathrm{a}) \underline{\mu}=\mu \frac{-v}{\underline{v}}(\mathrm{t}-\mathrm{a}) \stackrel{\mu+\nu}{\underline{\mu}}, \text { for } \mathrm{t} \in \mathbb{N}_{\mathrm{a}+\mu+v},
$$

and

$$
\Delta_{a+\mu}^{v}(t-a) \underline{\mu}=\mu^{\underline{v}}(t-a) \stackrel{\mu-v}{\underline{\mu}}, \text { for } t \in \mathbb{N}_{a+\mu+N-v} \text {. }
$$

Proof. It is easy to show (3.1) using the definition of the delta difference and properties of the gamma function. For (3.2) and (3.3) , first notice that $(t-a) \underline{\mu},(t-a) \frac{\mu+v}{a n d}(t-a) \frac{\mu-v}{a r e}$ each well-defined and positive on their respective domains $\mathbb{N}_{a+\mu}, \mathbb{N}_{a+\mu+v}$ and $\mathbb{N}_{a+\mu+N-v}$.

To prove (3.2), we suppose $\mu \in \mathbb{R} \backslash(-\mathbb{N})$ and consider the two cases $v=1$ and $v \in(0,1) \cup(1, \infty)$ 
separately. For $v=1$, we see from direct calculation that

$$
\begin{aligned}
\Delta_{a-\mu}^{-1}(t-a) \underline{\mu} & =\Delta_{a-\mu}^{-1} \Delta\left(\frac{(t-a) \frac{\mu+1}{\mu+1}}{\mu+1}\right), u \operatorname{sing}(3.1) \\
& =\sum_{s=a+\mu}^{t-1}\left(\frac{(s+1-a) \frac{\mu+1}{\mu+1}}{\mu+1}-\frac{(s-a) \frac{\mu+1}{\mu+1}}{\mu+1}\right) \\
& =\frac{(t-a) \frac{\mu+1}{\mu+1}-\frac{\mu \frac{\mu+1}{\mu+1}}{\mu+1}}{} \\
& =\mu \frac{-1}{-}(t-a) \frac{\mu+1}{}
\end{aligned}
$$

For $v>0$ with $v \neq 1$, define

$$
\begin{aligned}
& g_{1}(t):=\Delta_{a+\mu}^{-v}(t-a) \underline{\mu}=\frac{1}{\Gamma(\nu)} \sum_{s=a+\mu}^{t-v}(t-\sigma(s)) \frac{v-1}{(s-a) \underline{\mu}} \text { and } \\
& g_{2}(t):=\mu \frac{-v}{}(t-a) \stackrel{\mu+\nu}{ } \text {, }
\end{aligned}
$$

each for $t \in \mathbb{N}_{a+\mu+v}$. We will show that both $g_{1}$ and $g_{2}$ solve the first-order initial value problem

$$
\left\{\begin{array}{l}
(t-a-(\mu+v)+1) \Delta g(t)=(\mu+v) g(t), \text { for } t \in \mathbb{N}_{a+\mu+v} \\
g(a+\mu+v)=\Gamma(\mu+1)
\end{array} .\right.
$$

Since

$$
\begin{aligned}
g_{1}(a+\mu+v) & =\frac{1}{\Gamma(v)} \sum_{s=a+\mu}^{a+\mu}(a+\mu+v-\sigma(s)) \frac{v-1}{(s-a) \underline{\mu}} \\
& =\frac{1}{\Gamma(v)}(v-1) \frac{v-1}{\mu} \mu^{\underline{\mu}} \\
& =\Gamma(\mu+1),
\end{aligned}
$$

and

$$
\begin{aligned}
g_{2}(a+\mu+v) & =\mu \frac{-v}{\underline{a}}(\mu+v) \underline{\mu+v} \\
& =\frac{\Gamma(\mu+1)}{\Gamma(\mu+1+v)} \Gamma(\mu+v+1) \\
& =\Gamma(\mu+1),
\end{aligned}
$$

both $g_{1}$ and $g_{2}$ satisfy the initial condition in (3.4). 
We next show that $g_{1}$ satisfies the difference equation in (3.4). For $t \in \mathbb{N}_{a+\mu+\nu}$,

$$
\begin{aligned}
& \Delta \mathrm{g}_{1}(\mathrm{t})=\Delta\left[\frac{1}{\Gamma(v)} \sum_{s=\mathrm{a}+\mu}^{\mathrm{t}-\boldsymbol{v}}(\mathrm{t}-\sigma(\mathrm{s})) \underline{v-1}(\mathrm{~s}-\mathrm{a})^{\underline{\mu}}\right] \quad(\operatorname{apply}(2.1)) \\
& =\frac{1}{\Gamma(v)} \sum_{s=a+\mu}^{t-v}(v-1)(t-\sigma(s)) \frac{v-2}{(s-a) \underline{\mu}} \\
& +\frac{(t+1-(t+2-v)) \underline{v-1}}{\Gamma(v)}(t+1-v-a) \underline{\mu} \\
& =\frac{v-1}{\Gamma(v)} \sum_{s=a+\mu}^{t-v}(t-\sigma(s)) \frac{v-2}{}(s-a) \underline{\mu}+(t+1-v-a)^{\underline{\mu}} .
\end{aligned}
$$

Also, we may manipulate $g_{1}$ directly:

$$
\begin{aligned}
& g_{1}(t) \\
& =\frac{1}{\Gamma(v)} \sum_{s=a+\mu}^{t-v}(t-\sigma(s)) \frac{v-1}{(s-a) \underline{\mu}} \\
& =\frac{1}{\Gamma(v)} \sum_{s=a+\mu}^{t-\gamma}(t-\sigma(s)-(v-2))(t-\sigma(s)) \frac{v-2}{(s-a)} \underline{\mu} \\
& =\frac{1}{\Gamma(\nu)} \sum_{s=a+\mu}^{t-v}[(t-a-(\mu+v)+1)-(s-a-\mu)](t-\sigma(s)) \frac{v-2}{(s-a) \underline{\mu}} \\
& =\frac{\mathrm{t}-\mathrm{a}-(\mu+v)+1}{\Gamma(v)} \sum_{s=a+\mu}^{t-\gamma}(t-\sigma(s)) \frac{v-2}{(s-a) \underline{\mu}} \\
& -\frac{1}{\Gamma(v)} \sum_{s=a+\mu}^{t-v}(t-\sigma(s)) \frac{v-2}{(s-a)}(\underline{\mu+1} \\
& =h(t)-k(t),
\end{aligned}
$$

where

$$
\left\{\begin{array}{l}
h(t):=\frac{t-a-(\mu+v)+1}{\Gamma(\nu)} \sum_{s=a+\mu}^{t-\gamma}(t-\sigma(s)) \frac{v-2}{s}(s-a) \underline{\mu} \\
k(t):=\frac{1}{\Gamma(v)} \sum_{s=a+\mu}^{t-v}(t-\sigma(s)) \frac{v-2}{(s-a) \frac{\mu+1}{}}
\end{array} .\right.
$$

Integrating $k$ by parts,

$$
\begin{aligned}
k(t) & =\frac{1}{\Gamma(v)}\left[\sum_{s=a+\mu}^{(t-v+1)-1}(s-a) \frac{\mu+1}{v} \Delta\left(-\frac{(t-s) \frac{v-1}{v-1}}{v-1}\right]\right. \\
& =\frac{1}{\Gamma(v)}\left[\left(\left.(s-a) \frac{\mu+1}{s}\left(-\frac{(t-s) \frac{v-1}{v-1}}{v-1}\right)\right|_{s=a+\mu} ^{s=t-v+1}\right.\right.
\end{aligned}
$$




$$
\begin{aligned}
& \left.-\sum_{s=a+\mu}^{(t-v+1)-1}\left(-\frac{(t-\sigma(s))^{\frac{v-1}{}}}{v-1}(\mu+1)(s-a)^{\mu}\right)\right] \\
& =\frac{1}{\Gamma(v)}\left[-\frac{\Gamma(v)}{v-1}(t-v+1-a) \frac{\mu+1}{\underline{n}}\right. \\
& \left.+\frac{\mu+1}{v-1} \sum_{s=a+\mu}^{t-\gamma}(t-\sigma(s)) \frac{v-1}{(s-a)} \underline{\mu}\right] \\
& =\frac{1}{v-1}\left[\frac{\mu+1}{\Gamma(v)} \sum_{s=a+\mu}^{t-v}(t-\sigma(s)) \frac{v-1}{v}(s-a) \underline{\mu}-(t-v+1-a) \frac{\mu+1}{]}\right] \text {. }
\end{aligned}
$$

It follows from the above work that

- $(t-a-(\mu+v)+1) \Delta g_{1}(t)=(v-1) h(t)+(t+1-v-a) \underline{\mu+1}$

- $(\mu+1) g_{1}(t)-(v-1) k(t)=(t+1-v-a) \stackrel{\mu+1}{\text {. }}$

Hence,

$$
\begin{aligned}
(t-a-(\mu+v)+1) \Delta g_{1}(t) & =(v-1) h(t)+(\mu+1) g_{1}(t)-(v-1) k(t) \\
& =(v-1) g_{1}(t)+(\mu+1) g_{1}(t) \\
& =(\mu+v) g_{1}(t) .
\end{aligned}
$$

Finally, $g_{2}$ also satisfies the difference equation in (3.4):

$$
\begin{aligned}
& (t-a-(\mu+v)+1) \Delta g_{2}(t) \\
& =(t-a-(\mu+v)+1)\left[\mu \frac{-v}{}(\mu+v)(t-a) \frac{\mu+v-1}{\mu}\right], \text { by } \\
& =(\mu+1) \\
& =(\mu+v) \mu^{-v}[(t-a-(\mu+v-1))(t-a) \underline{\mu+v-1}] \\
& =(\mu+v) g_{2}(t) .
\end{aligned}
$$

By the uniqueness of solutions to initial value problems for first order difference operators, we conclude that $g_{1} \equiv g_{2}$ on $\mathbb{N}_{a+\mu+v}$.

We employ (3.1) and (3.2) in showing (3.3) as follows: For $t \in \mathbb{N}_{\mathbf{a}+\mu+\mathrm{N}-\nu}$,

$$
\begin{aligned}
& \Delta_{a+\mu}^{v}(t-a) \underline{\mu} \\
& =\Delta^{N}\left[\Delta_{a+\mu}^{-(N-v)}(t-a) \underline{\mu}\right] \\
& =\Delta^{N}\left[\frac{\Gamma(\mu+1)}{\Gamma(\mu+1+N-v)}(t-a) \underline{\mu+N-v}\right], \text { by (3.2) } \\
& =\frac{\Gamma(\mu+1)}{\Gamma(\mu+1+N-v)}((\mu+N-v) \cdots(\mu+1-v))(t-a) \frac{\mu-v}{n}, \text { by (3.1) }
\end{aligned}
$$




$$
\begin{aligned}
& =\frac{\Gamma(\mu+1)}{\Gamma(\mu+1+N-v)} \frac{\Gamma(\mu+N-v+1)}{\Gamma(\mu+1-v)}(t-a) \underline{\mu-v} \\
& =\mu^{\underline{v}}(t-a) \underline{\mu-v} .
\end{aligned}
$$

In the special case $v \in\{\mu+1, \mu+2, \ldots\}$, we have $\mu+1-v \in\left(-\mathbb{N}_{0}\right)$, and so

$$
\mu^{\underline{v}}=\frac{\Gamma(\mu+1)}{\Gamma(\mu+1-v)}
$$

from (3.3) is not well-defined. In this case, we interpret the right hand side of (3.3) as zero, which is as we desire.

\subsection{Composing a Sum with a Sum}

The rule for composing two fractional sums depends on an appropriate application of power rule (3.2) presented in Lemma 3.1 (see [1]).

Theorem 3.1. Let $\mathrm{f}: \mathbb{N}_{\mathbf{a}} \rightarrow \mathbb{R}$ be given and suppose $\boldsymbol{\gamma}, \mu>0$. Then

$$
\Delta_{\mathrm{a}+\mu}^{-\nu} \Delta_{\mathrm{a}}^{-\mu} \mathrm{f}(\mathrm{t})=\Delta_{\mathrm{a}}^{-\nu-\mu} \mathrm{f}(\mathrm{t})=\Delta_{\mathrm{a}+\nu}^{-\mu} \Delta_{\mathrm{a}}^{-\nu} \mathrm{f}(\mathrm{t}), \text { for } \mathrm{t} \in \mathbb{N}_{\mathrm{a}+\mu+\nu} .
$$

Proof. Suppose $f: \mathbb{N}_{a} \rightarrow \mathbb{R}$ and $\nu, \mu>0$. Then for $t \in \mathbb{N}_{a+\mu+v}$,

$$
\begin{aligned}
\Delta_{a+\mu}^{-v} \Delta_{a}^{-\mu} f(t) & =\frac{1}{\Gamma(v)} \sum_{s=a+\mu}^{t-v}(t-\sigma(s)) \frac{v-1}{\Gamma}\left(\frac{1}{\Gamma(\mu)} \sum_{r=a}^{s-\mu}(s-\sigma(r)) \frac{\mu-1}{f} f(r)\right) \\
& =\frac{1}{\Gamma(v) \Gamma(\mu)} \sum_{s=a+\mu} \sum_{r=a}^{s-\mu}(t-\sigma(s)) \frac{v-1}{v}(s-\sigma(r)) \frac{\mu-1}{f} f(r) \\
& =\frac{1}{\Gamma(v) \Gamma(\mu)} \sum_{r=a}^{t-(v+\mu)} \sum_{s=r+\mu}^{t-v}(t-\sigma(s)) \frac{v-1}{s}(s-\sigma(r)) \frac{\mu-1}{f} f(r) .
\end{aligned}
$$

Letting $x=s-\sigma(r)$, we continue:

$$
\begin{aligned}
& =\frac{1}{\Gamma(\nu) \Gamma(\mu)} \sum_{r=a}^{t-(\nu+\mu)}\left[\sum_{x=\mu-1}^{t-v-r-1}(t-x-r-2) \frac{v-1}{\nu} x^{\mu-1}\right] f(r) \\
& =\frac{1}{\Gamma(\mu)} \sum_{r=a}^{t-(v+\mu)}\left[\frac{1}{\Gamma(\nu)} \sum_{x=\mu-1}^{(t-r-1)-\nu}((t-r-1)-\sigma(x)) \frac{\nu-1}{2} x^{\mu-1}\right] f(r) \\
& =\frac{1}{\Gamma(\mu)} \sum_{r=a}^{t-(\nu+\mu)}\left(\left.\left[\Delta_{\mu-1}^{-\nu}\left(\frac{t-1}{\mu-1}\right)\right]\right|_{t-r-1} f(r)\right)
\end{aligned}
$$

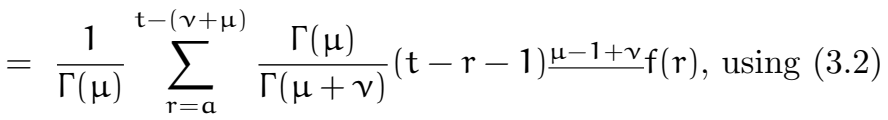




$$
\begin{aligned}
& =\frac{1}{\Gamma(\nu+\mu)} \sum_{r=a}^{t-(\nu+\mu)}(t-\sigma(r)) \frac{(\nu+\mu)-1}{f} f(r) \\
& =\Delta_{a}^{-(\nu+\mu)} f(t) \\
& =\Delta_{a}^{-\nu-\mu} f(t) .
\end{aligned}
$$

Since $\nu$ and $\mu$ are arbitrary, we conclude more generally

$$
\Delta_{\mathrm{a}+\mu}^{-v} \Delta_{\mathrm{a}}^{-\mu} \mathrm{f}(\mathrm{t})=\Delta_{\mathrm{a}}^{-v-\mu} \mathrm{f}(\mathrm{t})=\Delta_{\mathrm{a}+v}^{-\mu} \Delta_{\mathrm{a}}^{-v} \mathrm{f}(\mathrm{t}), \text { for } \mathrm{t} \in \mathbb{N}_{\mathrm{a}+v+\mu}
$$

Remark 3.1. In applying (3.2) above, we are allowed to write

$$
\Delta_{\mu-1}^{-v}[\tau \stackrel{\mu-1}{\underline{\mu}}]=\frac{\Gamma(\mu)}{\Gamma(\mu+\nu)} \tau \stackrel{\mu-1+v}{ }, \text { for } \tau \in \mathbb{N}_{\mu-1+\nu} .
$$

Since we are working with

$$
\mathrm{t} \in \mathbb{N}_{\mathrm{a}+\mu+v} \text { and } \mathrm{r} \in\{\mathrm{a}, \ldots, \mathrm{t}-\mu-v\},
$$

it is indeed appropriate to evaluate these terms at $\mathrm{t}-\mathrm{r}-1 \in \mathbb{N}_{\mu+v-1}$.

\subsection{Composing a Difference with a Sum}

Before studying the more general composition $\Delta_{\mathrm{a}+\mu}^{\nu} \circ \Delta_{\mathrm{a}}^{-\mu}$, we first consider the special case when $v \in \mathbb{N}_{0}$. Note that Atici and Eloe [2] show (3.5) below for the case $\mu>k$.

Lemma 3.2. Let $\mathrm{f}: \mathbb{N}_{\mathrm{a}} \rightarrow \mathbb{R}$ be given. For any $\mathrm{k} \in \mathbb{N}_{0}$ and $\mu>0$ with $M-1<\mu \leq M$, we have

$$
\begin{gathered}
\Delta^{k} \Delta_{a}^{-\mu} f(t)=\Delta_{a}^{k-\mu} f(t), \text { for } t \in \mathbb{N}_{a+\mu} \\
\Delta^{k} \Delta_{a}^{\mu} f(t)=\Delta_{a}^{k+\mu} f(t), \text { for } t \in \mathbb{N}_{a+M-\mu}
\end{gathered}
$$

Proof. Let $\mathrm{f}, \mu, \mathrm{M}$ and $\mathrm{k}$ be given as in the statement of the lemma.

Case 3.1. $(\mu=M)$

Observe that for $t \in \mathbb{N}_{a+1}$,

$$
\Delta \Delta_{a}^{-1} f(t)=\Delta\left[\sum_{s=a}^{t-1} f(s)\right]=\sum_{s=a}^{t} f(s)-\sum_{s=a}^{t-1} f(s)=f(t) .
$$

Likewise, for any $k \in \mathbb{N}$,

$$
\begin{aligned}
\Delta^{k} \Delta_{a}^{-k} f(t) & =\Delta^{k-1}\left[\Delta \Delta_{a+k-1}^{-1}\left(\Delta_{a}^{-(k-1)} f(t)\right)\right] \\
& =\Delta^{k-1} \Delta_{a}^{-(k-1)} f(t) \\
& =\cdots \cdots \\
& =f(t), \text { for } t \in \mathbb{N}_{a+k} .
\end{aligned}
$$


So, for any $t \in \mathbb{N}_{a+M}$,

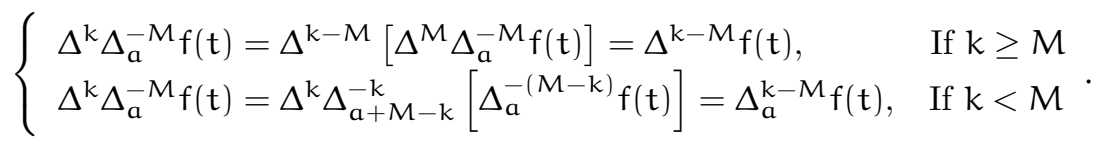

For (3.6), it is clear already that whole order difference operators commute.

Case 3.2. $(M-1<\mu<M)$

We first show that $\Delta \Delta_{\mathrm{a}}^{\mu} \mathrm{f}(\mathrm{t})=\Delta_{\mathrm{a}}^{1+\mu} \mathrm{f}(\mathrm{t})$, for $\mathrm{t} \in \mathbb{N}_{\mathrm{a}+\mathrm{M}-\mu}$.

Given $\mathrm{t} \in \mathbb{N}_{\mathrm{a}+\mathrm{M}-\mu}$ and using Definition 2.1 for $\Delta_{\mathrm{a}}^{\mu} \mathrm{f}$, we find

$$
\begin{aligned}
& \Delta \Delta_{a}^{\mu} f(t) \\
& \left.=\Delta\left[\frac{1}{\Gamma(-\mu)} \sum_{s=a}^{t+\mu}(t-\sigma(s)) \frac{-\mu-1}{f} f(s)\right](\operatorname{apply} \sqrt{2.1})\right) \\
& =\frac{1}{\Gamma(-\mu)} \sum_{s=a}^{t+\mu}(-\mu-1)(t-\sigma(s)) \frac{-\mu-2}{f} f(s)+f(t+\mu+1) \\
& =\frac{1}{\Gamma(-\mu-1)} \sum_{s=a}^{t+\mu}(t-\sigma(s)) \frac{-\mu-2}{f} f(s)+f(t+\mu+1) \\
& =\frac{1}{\Gamma(-\mu-1)} \sum_{s=a}^{t+\mu+1}(t-\sigma(s)) \frac{-\mu-2}{t} f(s) \\
& =\frac{1}{\Gamma(-\mu-1)} \sum_{s=a}(t-\sigma(s)) \underline{(-\mu-1)-1} f(s) \\
& =\Delta_{a}^{-(-\mu-1)} f(t) \\
& =\Delta_{a}^{1+\mu} f(t) . \quad
\end{aligned}
$$

Therefore, for any $\mathrm{k} \in \mathbb{N}$,

$$
\begin{aligned}
\Delta^{k} \Delta_{a}^{\mu} f(t) & =\Delta^{k-1}\left[\Delta \Delta_{a}^{\mu} f(t)\right] \\
& =\Delta^{k-1} \Delta_{a}^{1+\mu} f(t) \\
& =\cdots \cdots \\
& =\Delta_{a}^{k+\mu} f(t), \text { for } t \in \mathbb{N}_{a+M-\mu} \text {, proving }
\end{aligned}
$$

The results presented in Section 2 allow us to employ identical work as shown above to prove (3.5), since we may replace each $\mu$ above with a negative $\mu$.

We now have all the tools in hand to write down a rule for composing fractional differences with fractional sums. 
Theorem 3.2. Let $\mathrm{f}: \mathbb{N}_{\mathrm{a}} \rightarrow \mathbb{R}$ be given, and suppose $\boldsymbol{v}, \mu>0$ with $\mathrm{N}-1<v \leq \mathrm{N}$. Then

$$
\Delta_{\mathrm{a}+\mu}^{v} \Delta_{\mathrm{a}}^{-\mu} \mathrm{f}(\mathrm{t})=\Delta_{\mathrm{a}}^{v-\mu} \mathrm{f}(\mathrm{t}), \text { for } \mathrm{t} \in \mathbb{N}_{\mathrm{a}+\mu+\mathrm{N}-v} .
$$

Proof. Given $f, v, N$ and $\mu$ as in the statement of the theorem and $t \in \mathbb{N}_{a+\mu+\mathbb{N}-v}$,

$$
\begin{aligned}
\Delta_{a+\mu}^{v} \Delta_{a}^{-\mu} f(t) & =\Delta^{N} \Delta_{a+\mu}^{-(N-v)} \Delta_{a}^{-\mu} f(t) \\
& =\Delta^{N} \Delta_{a}^{-(N-v+\mu)} f(t), \text { using Theorem 3.1. } \\
& =\Delta_{a}^{N-(N-v+\mu)} f(t), \text { using Lemma 3.2. } \\
& =\Delta_{a}^{v-\mu} f(t) .
\end{aligned}
$$

Remark 3.2. One may wonder if the correct domain has been chosen in Theorem 3.2. To check this, we write out

$$
\left\{\begin{array}{l}
\mathcal{D}\left\{\Delta_{\mathrm{a}+\mu}^{v} \Delta_{\mathrm{a}}^{-\mu} \mathrm{f}\right\}=\mathbb{N}_{\mathrm{a}+\mu+\mathrm{N}-v} \\
\mathcal{D}\left\{\Delta_{\mathrm{a}}^{v-\mu} \mathrm{f}(\mathrm{t})\right\}=\mathbb{N}_{\mathrm{a}+\mu-v}, \text { if } v<\mu \\
\mathcal{D}\left\{\Delta_{\mathrm{a}}^{v-\mu} \mathrm{f}(\mathrm{t})\right\}=\mathbb{N}_{\mathrm{a}+\lceil\nu-\mu\rceil-(\nu-\mu)}, \text { if } v \geq \mu
\end{array},\right.
$$

and notice that in both cases, $\mathcal{D}\left\{\Delta_{\mathrm{a}+\mu}^{\nu} \Delta_{\mathrm{a}}^{-\mu} \mathrm{f}\right\} \subseteq \mathcal{D}\left\{\Delta_{\mathrm{a}}^{v-\mu} \mathrm{f}(\mathrm{t})\right\}$. We reason that the composition rule holds on $\mathbb{N}_{\mathbf{a}+\mu+\mathrm{N}-\boldsymbol{v}}$, the intersection of the domains of the left and right hand sides of the equation.

\subsection{Composing a Sum with a Difference}

For the remaining two composition rules - those whose inner operation is differentiation-we may not simply add the two operators' orders. This comes as no surprise, however, in light of the Fundamental Theorem of Calculus:

$$
\sum_{s=a}^{t-1}(\Delta f(s))=f(t)-f(a)
$$

Theorem 3.3. Let $\mathrm{f}: \mathbb{N}_{\mathrm{a}} \rightarrow \mathbb{R}$ be given, and suppose $\mathrm{k} \in \mathbb{N}_{0}$ and $\boldsymbol{\nu}, \mu>0$ with $M-1<\mu \leq$ M. Then,

$$
\Delta_{a}^{-v} \Delta^{k} f(t)=\Delta_{a}^{k-v} f(t)-\sum_{j=0}^{k-1} \frac{\Delta^{j} f(a)}{\Gamma(v-k+j+1)}(t-a) \frac{v-k+j}{},
$$

for $\mathrm{t} \in \mathbb{N}_{\mathrm{a}+\boldsymbol{v}}$, and

$$
\begin{aligned}
& \Delta_{a+M-\mu}^{-v} \Delta_{a}^{\mu} f(t)= \\
& \Delta_{a}^{\mu-v} f(t)-\sum_{j=0}^{M-1} \frac{\Delta_{a}^{j-(M-\mu)} f(a+M-\mu)}{\Gamma(\nu-M+j+1)}(t-a-M+\mu) \frac{v-M+j}{2}
\end{aligned}
$$

for $\mathrm{t} \in \mathbb{N}_{\mathrm{a}+\mathrm{M}-\mu+\boldsymbol{v}}$ 
Proof. Although (3.7) is merely a special case of (3.8), it is significant in its own right, and its proof (found in 2]) provides a stepping stone to (3.8).

(3.7) Let $k \in \mathbb{N}_{0}$ be given, and suppose $v>0$ with $v \notin\{1,2, \ldots, k-1\}$. Then summing by parts with $t \in \mathbb{N}_{a+v}$,

$$
\begin{aligned}
& \Delta_{\mathrm{a}}^{-v} \Delta^{\mathrm{k}} \mathrm{f}(\mathrm{t})
\end{aligned}
$$

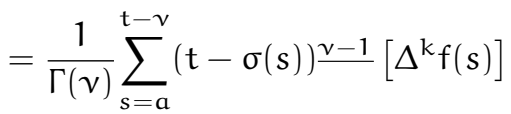

$$
\begin{aligned}
& =\frac{1}{\Gamma(v)} \sum_{s=a}^{(t-v+1)-1}(t-\sigma(s)) \frac{v-1}{\Delta} \Delta\left(\Delta^{k-1} f(s)\right), \\
& =\frac{1}{\Gamma(v)}\left[\left.(t-s) \frac{v-1}{\Delta^{k-1} f(s)}\right|_{s=a} ^{s=t-v+1}\right. \\
& -\sum_{s=a}^{t-v}\left(-(v-1)(t-\sigma(s)) \frac{v-2}{\left.\left.\Delta^{k-1} f(s)\right)\right]}\right. \\
& =\Delta^{k-1} f(t-v+1)-\frac{(t-a) \frac{v-1}{\Gamma(v)}}{k-1} f(a) \\
& +\frac{1}{\Gamma(v-1)} \sum_{s=a}^{t-v}\left((t-\sigma(s)) \frac{v-2}{\Delta^{k-1} f(s)}\right)
\end{aligned}
$$

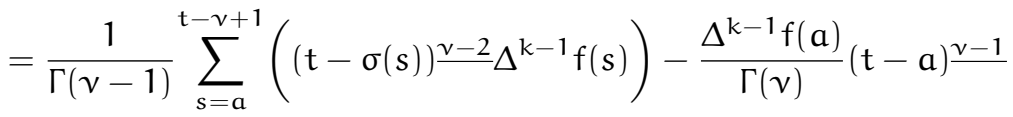

$$
\begin{aligned}
& =\Delta_{a}^{-(v-1)}\left[\Delta^{k-1} f(t)\right]-\frac{\Delta^{k-1} f(a)}{\Gamma(v)}(t-a) \frac{v-1}{} \\
& =\Delta_{a}^{1-v} \Delta^{k-1} f(t)-\frac{\Delta^{k-1} f(a)}{\Gamma(v)}(t-a) \frac{v-1}{} .
\end{aligned}
$$

Continuing summation by parts $(k-1)$-more times yields

$$
\begin{aligned}
& \Delta_{\mathrm{a}}^{-v} \Delta^{\mathrm{k}} f(\mathrm{t}) \\
& =\Delta_{a}^{1-v} \Delta^{k-1} f(t)-\frac{\Delta^{k-1} f(a)}{\Gamma(v)}(t-a) \frac{v-1}{} \\
& =\Delta_{a}^{2-v} \Delta^{k-2} f(t)-\frac{\Delta^{k-1} f(a)}{\Gamma(v)}(t-a) \frac{v-1}{\Gamma(v-1)}-\frac{\Delta^{k-2} f(a)}{(t-a) \frac{v-2}{2}} \\
& =\Delta_{a}^{k-v} f(t)-\sum_{i=1}^{k} \frac{\Delta^{k-i} f(a)}{\Gamma(v-i+1)}(t-a) \frac{v-i}{} \\
& =\Delta_{a}^{k-v} f(t)-\sum_{j=0}^{k-1} \frac{\Delta^{j} f(a)}{\Gamma(v-k+j+1)}(t-a) \frac{v-k+j}{2} \text {, for } t \in \mathbb{N}_{a+v} \text {. }
\end{aligned}
$$


Note that our assumption $v \notin\{1,2, \ldots, k-1\}$ implies that $v-k+j+1 \notin\left(-\mathbb{N}_{0}\right)$, and so the above expression is well-defined.

Next, suppose that $v \in\{1,2, \ldots, k-1\}$. Then $k-v \in \mathbb{N}$, and so for $t \in \mathbb{N}_{a+v}$,

$$
\begin{aligned}
& \Delta_{a}^{-v} \Delta^{k} f(t) \\
& =\Delta^{k-v} \Delta_{a+v}^{-(k-v)} \Delta_{a}^{-v} \Delta^{k} f(t), \text { using Theorem 3.2 } \\
& =\Delta^{k-v}\left[\Delta_{a}^{-k} \Delta^{k} f(t)\right], \text { using Theorem 3.1] } \\
& =\Delta^{k-v}\left[f(t)-\sum_{j=0}^{k-1} \frac{\Delta^{j} f(a)}{\Gamma(j+1)}(t-a) \underline{j}\right] \text {, applying the previous case } \\
& =\Delta^{k-v} f(t)-\sum_{j=0}^{k-1} \frac{\Delta^{j} f(a)}{\Gamma(j+1)}\left[\Delta^{k-v}(t-a) \underline{j}\right] \\
& =\Delta^{k-v} f(t)-\sum_{j=k-v}^{k-1} \frac{\Delta^{j} f(a)}{\Gamma(j+1)} \frac{\Gamma(j+1)}{\Gamma(j+1-k+v)}(t-a) \frac{j-k+v}{-}, \\
& =\Delta^{k-v} f(t)-\sum_{j=0}^{k-1} \frac{\Delta^{j} f(a)}{\Gamma(j+1-k+v)}(t-a) \frac{j-k+v}{n},
\end{aligned}
$$

since $\frac{1}{\Gamma(v+1-\mathrm{k})}=\cdots=\frac{1}{\Gamma(-1)}=\frac{1}{\Gamma(0)}=0$, by convention. The above two cases prove (3.7).

(3.8) Suppose now that $\gamma, \mu>0$ with $M-1<\mu \leq M$. Defining $g(t):=\Delta_{a}^{-(M-\mu)} f(t)$ and $b:=a+M-\mu$ (the first point in the domain of $g$ ), we have for $t \in \mathbb{N}_{a+M-\mu+v}$,

$$
\begin{aligned}
& \Delta_{a+M-\mu}^{-\nu} \Delta_{a}^{\mu} f(t) \\
& =\Delta_{\mathrm{a}+\mathrm{M}_{-\mu}}^{-\gamma} \Delta^{\mathrm{M}}(\mathrm{g}(\mathrm{t})) \text {, and applying (3.7), } \\
& =\Delta_{a+M-\mu}^{M-v} g(t)-\sum_{j=0}^{M-1} \frac{\Delta^{j} g(b)}{\Gamma(v-M+j+1)}(t-b) \underline{v-M+j} \\
& =\Delta_{a+M-\mu}^{M-v} \Delta_{a}^{-(M-\mu)} f(t)-\sum_{j=0}^{M-1} \frac{\Delta^{j} \Delta_{a}^{-(M-\mu)} f(b)}{\Gamma(v-M+j+1)}(t-b) \underline{v-M+j} \\
& =\Delta_{a}^{\mu-v} f(t)-\sum_{j=0}^{M-1} \frac{\Delta_{a}^{j-M+\mu} f(a+M-\mu)}{\Gamma(v-M+j+1)}(t-a-M+\mu) \underline{v-M+j},
\end{aligned}
$$

where in this last step, we applied Theorem 3.2 twice.

Remark 3.3. - Theorem 3.2 allows us to write (3.8) in the equivalent form

$$
\begin{aligned}
& \Delta_{a+M-\mu}^{-v} \Delta_{a}^{\mu} f(t)= \\
& \Delta_{a+v}^{\mu} \Delta_{a}^{-v} f(t)-\sum_{j=0}^{M-1} \frac{\Delta_{a}^{j-(M-\mu)} f(a+M-\mu)}{\Gamma(v-M+j+1)}(t-a-M+\mu) \underline{v-M+j},
\end{aligned}
$$


for $\mathrm{t} \in \mathbb{N}_{\mathrm{a}+\mathrm{M}-\mu+v}$.

- When $0<\mu \leq 1$, the term $\Delta_{\mathrm{a}}^{j-(\mathrm{M}-\mu)} \mathrm{f}(\mathrm{a}+\mathrm{M}-\mu)$ in (3.8) simplifies nicely to $\mathrm{f}(\mathrm{a})$. More generally for any $M-1<\mu \leq M$, we have that for $j \in\{0, \ldots, M-1\}$,

$$
\begin{aligned}
& \Delta_{a}^{j-(M-\mu)} f(a+M-\mu) \\
& =\left.\frac{1}{\Gamma(M-\mu-j)} \sum_{s=a}^{t+j-(M-\mu)}(t-\sigma(s)) \frac{M-\mu-j-1}{d} f(s)\right|_{t=a+M-\mu} \\
& =\frac{1}{\Gamma(M-\mu-j)} \sum_{s=a}^{a+j}(a+M-\mu-\sigma(s)) \frac{M-\mu-j-1}{d} f(s) \\
& =\frac{1}{\Gamma(M-\mu-j)} \sum_{k=0}^{j}(M-\mu-\sigma(k)) \frac{M-\mu-j-1}{\frac{M-1}{j}} f(k+a) \\
& =\sum_{k=0}^{j}\left(\begin{array}{c}
M-\mu-\sigma(k) \\
j-k
\end{array}\right) f(k+a) .
\end{aligned}
$$

\subsection{Composing a Difference with a Difference}

We conclude this section with a rule for composing two fractional differences. One quickly observes the similarity between the composition rule (3.9) below and the rule for $\Delta_{\mathbf{a}+M-\mu}^{-\gamma} \circ \Delta_{\mathfrak{a}}^{\mu}$ given in (3.8). The special case $\mu \in \mathbb{N}_{0}$ in (3.9) below was developed by Atici and Eloe in 2 - we extend their result here to the more general case $\mu>0$.

Theorem 3.4. Let $f: \mathbb{N}_{a} \rightarrow \mathbb{R}$ be given and suppose $v, \mu>0$ with $N-1<\nu \leq N$ and $\mathrm{M}-1<\mu \leq \mathrm{M}$. Then for $\mathrm{t} \in \mathbb{N}_{\mathrm{a}+\mathrm{M}-\mu+\mathrm{N}-\gamma}$,

$$
\begin{aligned}
& \Delta_{a+M-\mu}^{v} \Delta_{a}^{\mu} f(t)= \\
& \Delta_{a}^{v+\mu} f(t)-\sum_{j=0}^{M-1} \frac{\Delta_{a}^{j-M+\mu} f(a+M-\mu)}{\Gamma(-v-M+j+1)}(t-a-M+\mu) \underline{-v-M+j},
\end{aligned}
$$

where the terms in the summation vanish for $v \in \mathbb{N}_{0}$, by our convention for $\Gamma$.

Proof. Let $f, v$ and $\mu$ be given as in the statement of the theorem. Recall that Lemma 3.2 proves (3.9) in the case when $v=\mathrm{N}$. 


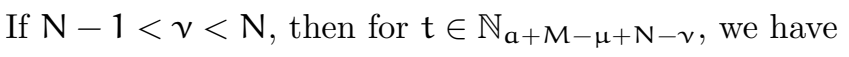

$$
\begin{aligned}
& \Delta_{\mathrm{a}+M-\mu}^{v} \Delta_{\mathrm{a}}^{\mu} \mathrm{f}(\mathrm{t}) \\
& =\Delta^{N}\left[\Delta_{a+M-\mu}^{-(N-v)} \Delta_{a}^{\mu} f(t)\right] \\
& =\Delta^{N}\left[\Delta_{a}^{-N+v+\mu} f(t)-\right. \\
& \sum_{j=0}^{M-1} \frac{\Delta_{a}^{j-M+\mu} f(a+M-\mu)}{\Gamma(N-v-M+j+1)}(t-a-M+\mu) \frac{N-v-M+j}{\Gamma(3.8)} \text {, by } \\
& =\Delta_{\mathrm{a}}^{v+\mu} \mathrm{f}(\mathrm{t})- \\
& \sum_{j=0}^{M-1} \frac{\Delta_{a}^{j-M+\mu} f(a+M-\mu)}{\Gamma(N-v-M+j+1)} \Delta^{N}\left[(t-a-M+\mu) \frac{N-v-M+j}{2}\right] \\
& =\Delta_{a}^{v+\mu} f(t)-\sum_{j=0}^{M-1} \frac{\Delta_{a}^{j-M+\mu} f(a+M-\mu)}{\Gamma(-v-M+j+1)}(t-a-M+\mu) \underline{-v-M+j} .
\end{aligned}
$$

By the same token as (3.9),

$$
\begin{aligned}
& \Delta_{a+N-v}^{\mu} \Delta_{a}^{v} f(t)= \\
& \Delta_{a}^{\mu+v} f(t)-\sum_{j=0}^{N-1} \frac{\Delta_{a}^{j-N+v} f(a+N-v)}{\Gamma(-\mu-N+j+1)}(t-a-N+v) \underline{-\mu-N+j},
\end{aligned}
$$

where the terms in the summation vanish for $\mu \in \mathbb{N}_{0}$. Combining this with (3.9), we may write two further rules for composing fractional differences:

Corollary 3.1. Let $\mathrm{f}: \mathbb{N}_{\mathrm{a}} \rightarrow \mathbb{R}$ and $\boldsymbol{v}, \mu>0$ be given, with $\mathrm{N}-1<v \leq \mathrm{N}$ and $\mathrm{M}-1<\mu \leq \mathrm{M}$. Then,

$$
\begin{aligned}
& \Delta_{a+M-\mu}^{\nu} \Delta_{a}^{\mu} f(t)=\Delta_{a+N-v}^{\mu} \Delta_{a}^{\nu} f(t) \\
& +\sum_{j=0}^{N-1} \frac{\Delta_{a}^{j-N+v} f(a+N-v)}{\Gamma(-\mu-N+j+1)}(t-a-N+v) \underline{-\mu-N+j} \\
& -\sum_{j=0}^{M-1} \frac{\Delta_{a}^{j-M+\mu} f(a+M-\mu)}{\Gamma(-v-M+j+1)}(t-a-M+\mu) \underline{-v-M+j},
\end{aligned}
$$

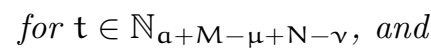

$$
\Delta_{a+N-v}^{v} \Delta_{a}^{v} f(t)=\Delta_{a}^{2 v} f(t)-\sum_{j=0}^{N-1} \frac{\Delta_{a}^{j-N+v} f(a+N-v)}{\Gamma(-v-N+j+1)}(t-a-N+v) \underline{-v-N+j}
$$

for $\mathrm{t} \in \mathbb{N}_{\mathrm{a}+2(\mathrm{~N}-v)}$. 


\section{Application and Examples}

To explicitly solve a nonhomogeneous, $v^{\text {th }}$-order fractional initial value problem, we need many of the tools developed thus far in Sections 2 and 3. Specifically, we apply the general power rule (3.3) in Lemma 3.1 and the two composition rules found in Theorems 3.2 and 3.3 ,

Theorem 4.1. Let $\mathrm{f}: \mathbb{N}_{\mathrm{a}} \rightarrow \mathbb{R}$ and $v>0$ be given, with $\mathrm{N}-1<v \leq \mathrm{N}$. Consider the $v^{\text {th }}$-order fractional difference equation

$$
\Delta_{a+v-N}^{v} y(t)=f(t), \quad t \in \mathbb{N}_{a},
$$

and the corresponding fractional initial value problem

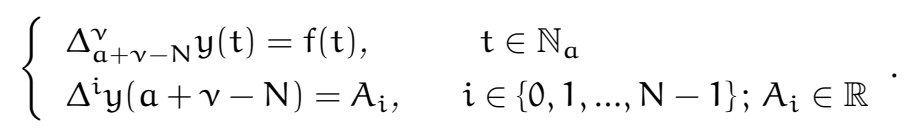

The general solution to 4.1) is given by

$$
y(t)=\sum_{i=0}^{N-1} \alpha_{i}(t-a) \frac{i+v-N}{d}+\Delta_{a}^{-v} f(t), \text { for } \alpha_{i} \in \mathbb{R}, t \in \mathbb{N}_{a+v-N},
$$

and the unique solution to 4.2) is

$$
y(t)=\sum_{i=0}^{N-1}\left(\sum_{p=0}^{i} \sum_{k=0}^{i-p} \frac{(-1)^{k}}{i !}(i-k) \frac{N-v}{i}\left(\begin{array}{c}
i \\
p
\end{array}\right)\left(\begin{array}{c}
i-p \\
k
\end{array}\right) A_{p}\right)(t-a) \frac{i+v-N}{d}+\Delta_{a}^{-v} f(t),
$$

for $\mathrm{t} \in \mathbb{N}_{\mathrm{a}+v-\mathrm{N}}$.

Proof. Let $f$ and $v$ be given as in the statement of the theorem. For arbitrary but fixed $\alpha_{i} \in \mathbb{R}$, define $y: \mathbb{N}_{a+v-N} \rightarrow \mathbb{R}$ by

$$
y(t):=\sum_{i=0}^{N-1} \alpha_{i}(t-a)^{i+v-N}+\Delta_{a}^{-v} f(t) .
$$

Here, we extend the usual domain of the fractional sum $\Delta_{\mathrm{a}}^{-v} \mathrm{f}$ to the larger set $\mathbb{N}_{\mathrm{a}+\boldsymbol{v}-\mathrm{N}}$ to include the $\mathrm{N}$ zeros of $\Delta_{\mathrm{a}}^{-v} \mathrm{f}$, as discussed in Section 1.3 , I.e.,

$$
\Delta_{a}^{-v} f(a+v-N)=\cdots=\Delta_{a}^{-v} f(a+v-1)=0 .
$$

To show that $y$ is the general solution of (4.1), we must show that any function of $y$ 's form is a solution to (4.1) and that every solution to (4.1) is of $y$ 's form. Beginning with the former, 
observe that for $t \in \mathbb{N}_{a}$,

$$
\begin{aligned}
& \Delta_{a+v-N}^{v} y(t) \\
& =\Delta_{a+v-N}^{v}\left[\sum_{i=0}^{N-1} \alpha_{i}(t-a) \frac{i+v-N}{d}+\Delta_{a}^{-v} f(t)\right] \\
& =\sum_{i=0}^{N-1} \alpha_{i} \Delta_{a+v-N}^{v}(t-a) \frac{i+v-N}{2}+\Delta_{a+v-N}^{v} \Delta_{a}^{-v} f(t) .
\end{aligned}
$$

At this point, we would like to apply the power rule (3.3) in the summation and Theorem 3.2 on the second term, but neither may be applied directly due to the mismatching lower limit on the operator $\Delta_{\mathrm{a}+\boldsymbol{v}-\mathrm{N}}^{v}$. However, this problem is quickly remedied by throwing away the zero terms involved:

$$
\begin{aligned}
\Delta_{a+v-N}^{v} \Delta_{a}^{-v} f(t) & =\frac{1}{\Gamma(-v)} \sum_{s=a+v-N}^{t+v}(t-\sigma(s)) \frac{-v-1}{\Delta_{a}^{-v}} \Delta_{a}^{-s}(s) \\
& =\frac{1}{\Gamma(-v)} \sum_{s=a+v}^{t+v}(t-\sigma(s)) \frac{-v-1}{-v} \Delta_{a}^{-v} f(s) \\
& =\Delta_{a+v}^{v} \Delta_{a}^{-v} f(t)
\end{aligned}
$$

and

$$
\begin{aligned}
& \Delta_{a+v-N}^{v}(t-a) \frac{i+v-N}{\Gamma(-v)} \sum_{s=a+v-N}^{t+v}(t-\sigma(s)) \frac{-v-1}{\Gamma}(s-a) \frac{i+v-N}{} \\
& =\frac{1}{\Gamma(-v)} \sum_{s=a+v-N+i}^{t+v}(t-\sigma(s)) \frac{-v-1}{(s-a)} \underline{i+v-N} \\
& =\Delta_{a+i+v-N}^{v}(t-a) \frac{i+v-N}{\text {. }}
\end{aligned}
$$

Therefore,

$$
\begin{aligned}
\Delta_{a+v-N}^{v} y(t) & =\sum_{i=0}^{N-1} \alpha_{i} \Delta_{a+i+v-N}^{v}(t-a) \frac{i+v-N}{d}+\Delta_{a+v}^{v} \Delta_{a}^{-v} f(t) \\
& =f(t), \text { applying Theorems 3.1 and 3.2 }
\end{aligned}
$$

Next, we show that every solution of (4.1) has y's form. Suppose that $z: \mathbb{N}_{\mathrm{a}+v-\mathrm{N}} \rightarrow \mathbb{R}$ is a solution to (4.1). Then we may apply Theorem 3.3 to solve (4.1) for $z$ :

$$
\begin{aligned}
& \Delta_{\mathrm{a}+v-\mathrm{N}}^{v} z(\mathrm{t})=\mathrm{f}(\mathrm{t}) \text {, for } \mathrm{t} \in \mathbb{N}_{\mathrm{a}} \\
& \Rightarrow \Delta_{\mathrm{a}}^{-v} \Delta_{\mathrm{a}+v-\mathrm{N}}^{v} z(\mathrm{t})=\Delta_{\mathrm{a}}^{-v} \mathrm{f}(\mathrm{t}) \text {, for } \mathrm{t} \in \mathbb{N}_{\mathrm{a}+v-\mathrm{N}} \\
& \Rightarrow \Delta_{a+v-N}^{0} z(t)-\sum_{i=0}^{N-1} \frac{\Delta_{a+v-N}^{i-(N-v)} z(a)}{\Gamma(v-N+i+1)}(t-a) \frac{v-N+i}{2}=\Delta_{a}^{-v} f(t)
\end{aligned}
$$




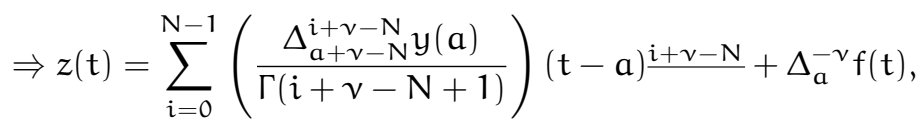

for $t \in \mathbb{N}_{a+v-N}$. As before, we have extended the domain of $\Delta_{a}^{-v} f$ to $\mathbb{N}_{a+v-N}$. Since $z$ has the same form as $y$, we have shown that

$$
y(t)=\sum_{i=0}^{N-1} \alpha_{i}(t-a) \frac{i+v-N}{d}+\Delta_{a}^{-v} f(t), t \in \mathbb{N}_{a+v-N}
$$

is the general solution of (4.1).

The next task is to find the particular $\alpha_{i} \in \mathbb{R}$ which make $y$ a solution to (4.2). From (4.3), it is clear that these constants have the form

$$
\alpha_{i}=\frac{\Delta_{a+v-N}^{i+v-N} y(a)}{\Gamma(i+v-N+1)}, i \in\{0, \ldots, N-1\},
$$

but we must write each $\alpha_{i}$ in terms of the initial conditions $\left\{A_{p}\right\}_{p=0}^{N-1}$. In other words, we must find a way to write each $\Delta_{a+v-N}^{i+v-N} y(a)$ in terms of $\left\{\Delta^{\mathrm{p}} \mathrm{y}(\mathrm{a}+\boldsymbol{v}-\mathrm{N})\right\}_{\mathrm{p}=0}^{\mathrm{N}-1}$. To accomplish this, we need

- the binomial representation of a fractional difference from Theorem 2.3 .

$$
\Delta_{a}^{v} g(t)=\sum_{k=0}^{t-a+v}(-1)^{k}\left(\begin{array}{l}
v \\
k
\end{array}\right) g(t+v-k), \text { for } t \in \mathbb{N}_{a+N-v}
$$

- the following well-known formula (see [6])

$$
g(t+m)=\sum_{k=0}^{m}\left(\begin{array}{l}
m \\
k
\end{array}\right) \Delta^{k} g(t), \text { for } m \in \mathbb{N}_{0} .
$$

Applying the above two facts directly yields

$$
\begin{aligned}
& \Delta_{a+v-N}^{i+v-N} y(a) \\
& =\sum_{k=0}^{i}(-1)^{k}\left(\begin{array}{c}
i+v-N) \\
k
\end{array}\right) y(a+i+v-N-k) \\
& =\sum_{k=0}^{i}(-1)^{k}\left(\begin{array}{c}
i+v-N \\
k
\end{array}\right) y((a+v-N)+(i-k)) \\
& =\sum_{k=0}^{i}(-1)^{k}\left(\begin{array}{c}
i+v-N \\
k
\end{array}\right) \sum_{p=0}^{i-k}\left(\begin{array}{c}
i-k \\
p
\end{array}\right) \Delta^{p} y(a+v-N) \\
& =\sum_{k=0}^{i} \sum_{p=0}^{i-k}(-1)^{k}\left(\begin{array}{c}
i+v-N \\
k
\end{array}\right)\left(\begin{array}{c}
i-k \\
p
\end{array}\right) A_{p}
\end{aligned}
$$




$$
\begin{aligned}
= & \sum_{p=0}^{i} \sum_{k=0}^{i-p}(-1)^{k}\left(\begin{array}{c}
i+v-N \\
k
\end{array}\right)\left(\begin{array}{c}
i-k \\
p
\end{array}\right) A_{p} \\
= & \sum_{p=0}^{i} \sum_{k=0}^{i-p}(-1)^{k} \frac{\Gamma(i+v-N+1)}{k ! \Gamma(i+v-N-k+1)} \frac{(i-k) !}{p !(i-k-p) !} A_{p} \\
= & \Gamma(i+v-N+1) \\
& \cdot \sum_{p=0}^{i} \sum_{k=0}^{i-p} \frac{(-1)^{k}}{i !} \frac{\Gamma(i-k+1)}{\Gamma(i-k+1+v-N)} \frac{i !}{p !(i-p) !} \frac{(i-p) !}{k !(i-p-k) !} A_{p} \\
= & \Gamma(i+v-N+1) \sum_{p=0}^{i} \sum_{k=0}^{i-p} \frac{(-1)^{k}}{i !}(i-k) \frac{N-v}{i}\left(\begin{array}{c}
i \\
p
\end{array}\right)\left(\begin{array}{c}
i-p \\
k
\end{array}\right) A_{p} .
\end{aligned}
$$

It follows that

$$
\alpha_{i}=\sum_{p=0}^{i}\left(\sum_{k=0}^{i-p} \frac{(-1)^{k}}{i !}(i-k) \frac{N-v}{i}\left(\begin{array}{c}
i-p \\
p
\end{array}\right)\right) A_{p}, \quad \text { for } i \in\{0, \ldots, N-1\} .
$$

Moreover, this solution is unique since the initial value problem (4.2) was solved directly, with no restricting assumptions or information lost.

When actually solving an initial value problem of the form (4.2), the constants $\alpha_{i}$ may seem cumbersome to calculate, especially by hand. However, we may write out more convenient and user-friendly expressions for the first several $\alpha_{i}$ for use in solving lower order problems. To do this, we define

$$
g: \mathbb{N}_{0} \rightarrow \mathbb{R} \text { by } g(t):=\mathrm{t} \frac{\mathrm{N}-\mathrm{v}}{} .
$$

Then the first five $\alpha_{i}$ (provided they are required) are given by:

$$
\begin{aligned}
\alpha_{0} & =g(0) A_{0}, \\
\alpha_{1} & =g(1) A_{1}-(g(0)-g(1)) A_{0}, \\
\alpha_{2} & =\frac{g(2)}{2} A_{2}-(g(1)-g(2)) A_{1}+\frac{g(0)-2 g(1)+g(2)}{2} A_{0}, \\
\alpha_{3} & =\frac{g(3)}{6} A_{3}-\frac{g(2)-g(3)}{2} A_{2}+\frac{g(1)-2 g(2)+g(3)}{2} A_{1} \\
& -\frac{g(0)-3 g(1)+3 g(2)-g(3)}{6} A_{0}, \\
\alpha_{4} & =\frac{g(4)}{24} A_{4}-\frac{g(3)-g(4)}{6} A_{3}+\frac{g(2)-2 g(3)+g(4)}{4} A_{2} \\
& -\frac{g(1)-3 g(2)+3 g(3)-g(4)}{6} A_{1} \\
& +\frac{g(0)-4 g(1)+6 g(2)-4 g(3)+g(4)}{24} A_{0} .
\end{aligned}
$$


Note that if $v=\mathrm{N}$ in (4.2), we are studying the well-known whole-order initial value problem

$$
\left\{\begin{array}{ll}
\Delta^{N} y(t)=f(t), & t \in \mathbb{N}_{a} \\
\Delta^{i} y(a)=A_{i}, & i \in\{0,1, \ldots, N-1\} ; A_{i} \in \mathbb{R}
\end{array} .\right.
$$

In this case, the solution given in Theorem 4.1 simplifies considerably:

$$
\begin{aligned}
y(t) & =\sum_{i=0}^{N-1}\left[\sum_{p=0}^{i}\left(\sum_{k=0}^{i-p} \frac{(-1)^{k}}{i !}\left(\begin{array}{c}
i \\
p
\end{array}\right)\left(\begin{array}{c}
i-p \\
k
\end{array}\right)\right) A_{p}\right](t-a)^{\underline{i}}+\Delta_{a}^{-N} f(t) \\
& =\sum_{i=0}^{N-1}\left[\sum_{p=0}^{i} \frac{A_{p}}{i !}\left(\begin{array}{l}
i \\
p
\end{array}\right) \sum_{k=0}^{i-p}(-1)^{k}\left(\begin{array}{c}
i-p \\
k
\end{array}\right)\right](t-a)^{i}+\Delta_{a}^{-N} f(t) \\
& =\sum_{i=0}^{N-1} \frac{A_{i}}{i !}(t-a)^{\underline{i}}+\Delta_{a}^{-N} f(t), \text { for } t \in \mathbb{N}_{a},
\end{aligned}
$$

since

$$
\sum_{k=0}^{i-p}(-1)^{k}\left(\begin{array}{c}
i-p \\
k
\end{array}\right)=\left\{\begin{array}{ll}
0, & i-p>0 \\
1, & i-p=0
\end{array} .\right.
$$

One may prefer to write everything in (4.5) in terms of $y$ :

$$
y(t)=\sum_{i=0}^{N-1} \frac{\Delta^{i} y(a)}{i !}(t-a)^{i}+\Delta_{a}^{-N} \Delta^{N} y(t),
$$

which produces a version of Taylor's Theorem for functions $y: \mathbb{N}_{a} \rightarrow \mathbb{R}$. In particular, since

$$
\Delta_{\mathrm{a}}^{-\mathrm{N}} \Delta^{\mathrm{N}} \mathrm{y}(\mathrm{t})=\frac{1}{\Gamma(\mathrm{N})} \sum_{s=\mathrm{a}}^{\mathrm{t}-\mathrm{N}}(\mathrm{t}-\sigma(\mathrm{s})) \frac{\mathrm{N}-1}{} \Delta^{\mathrm{N}} \mathrm{y}(\mathrm{s}) \rightarrow 0 \text { pointwise as } \mathrm{N} \rightarrow \infty,
$$

we may write

$$
y(t)=\sum_{i=0}^{\infty} \frac{\Delta^{i} y(a)}{i !}(t-a)^{i}, \text { for } t \in \mathbb{N}_{a}
$$

However, (4.6) turns out to be just another version of formula (4.4) given in the proof of Theorem 4.1. To see this, let $t \in \mathbb{N}_{a}$ be given by $t=a+m$, for some $m \in \mathbb{N}_{0}$. Then (4.6) becomes

$$
y(t)=y(a+m)=\sum_{i=0}^{\infty} \frac{\Delta^{i} y(a)}{i !} m^{i}=\sum_{i=0}^{m}\left(\begin{array}{c}
m \\
i
\end{array}\right) \Delta^{i} y(a)
$$

We close with two examples demonstrating ideas presented in this paper.

Example 4.1. $\quad$ Consider the following $2.7^{\text {th }}$-order initial value problem

$$
\left\{\begin{array}{l}
\Delta_{-0.3}^{2.7} y(t)=t^{\underline{2}}, t \in \mathbb{N}_{0} \\
y(-0.3)=2, \Delta y(-0.3)=3, \Delta^{2} y(-0.3)=5
\end{array} .\right.
$$


Note that (4.7) is a specific instance of (4.2) from Theorem 4.1, with

$$
\begin{array}{lll}
a=0, & v=2.7, \quad N=3, \quad f(t)=t^{2}, \\
A_{0}=2, & A_{1}=3, & A_{2}=5
\end{array}
$$

Therefore, the solution to (4.7) is given by

$$
\begin{aligned}
y(t) & =\sum_{i=0}^{N-1} \alpha_{i}(t-a) \frac{i+v-N}{d}+\Delta_{a}^{-v} f(t), \quad \text { for } t \in \mathbb{N}_{a+v-N} \\
& =\sum_{i=0}^{2} \alpha_{i} t^{i-0.3}+\Delta_{0}^{-2.7} t^{\underline{2}}, \quad \text { for } t \in \mathbb{N}_{-0.3,}
\end{aligned}
$$

where,

$$
\begin{aligned}
\alpha_{0} & =0 \frac{0.3}{2} A_{0} \\
\alpha_{1} & =1 \frac{0.3}{A_{1}}-\left(0 \frac{0.3}{2}-1 \frac{0.3}{2}\right) A_{0} \\
\alpha_{2} & =\frac{2 \frac{0.3}{2}}{2} A_{2}-\left(1 \frac{0.3}{2}-2 \frac{0.3}{)}\right) A_{1}+\frac{0 \frac{0.3}{2}-2 \cdot 1 \frac{0.3}{2}+2 \frac{0.3}{2} A_{0}}{2} \\
\alpha_{0} & \approx 1.541, \quad \alpha_{1} \approx 3.962, \quad \alpha_{2} \approx 3.684 .
\end{aligned}
$$

Our only remaining task is to calculate

$$
\begin{aligned}
\Delta_{0}^{-2.7} \mathrm{t}^{\underline{2}}=\frac{1}{\Gamma(2.7)} \sum_{\mathrm{s}=0}^{\mathrm{t}-2.7}(\mathrm{t}-\sigma(\mathrm{s})) \frac{1.7}{\mathrm{~s}} \mathrm{~s}^{\underline{2}} & =\frac{1}{\Gamma(2.7)} \sum_{\mathrm{s}=2}^{\mathrm{t}-2.7}(\mathrm{t}-\sigma(\mathrm{s})) \frac{1.7}{\mathrm{~s}} \mathrm{~s}^{\underline{2}} \\
& =\Delta_{2}^{-2.7} \mathrm{t}^{\underline{2}} \\
& =\frac{\Gamma(3)}{\Gamma(5.7)} \mathrm{t} \underline{4.7} \\
& \approx 0.0276 \mathrm{t} \underline{4.7}
\end{aligned}
$$

Therefore, the unique solution to (4.7) is

$$
y(t) \approx 1.541 \mathrm{t} \frac{-0.3}{-3.962 t \frac{0.7}{2}+3.684 t^{1.7}}+0.0276 t^{4.7}, \text { for } t \in \mathbb{N}_{-0.3} \text {. }
$$

Example 4.2. $\quad$ Consider the composed difference operator $\Delta_{\mathbf{a}+\mathbf{M}-\mu}^{\nu} \circ \Delta_{a}^{\mu}$, where $v$ and $\mu$ are two positive non-integers who sum to an integer. By how much does the fractional composition operator $\Delta_{\mathrm{a}+\mathrm{M}-\mu}^{\nu} \circ \Delta_{\mathrm{a}}^{\mu}$ differ from the whole-order operator $\Delta^{\nu+\mu}$ ?

Let $\mathrm{f}: \mathbb{N}_{\mathrm{a}} \rightarrow \mathbb{R}$ be given and choose $\mathrm{M}, \mathrm{N}, \mathrm{P} \in \mathbb{N}$ so that $\mathrm{N}-1<v<\mathrm{N}, \mathrm{M}-1<\mu<\mathrm{M}$ and $v+\mu=\mathrm{P}$. Then $\mathrm{N}+\mathrm{M}=\mathrm{P}+1$ and

$$
\mathcal{D}\left\{\Delta_{\mathrm{a}+\mathrm{M}-\mu}^{\nu} \Delta_{\mathrm{a}}^{\mu} f\right\}=\mathbb{N}_{\mathrm{a}+M-\mu+N-v}=\mathbb{N}_{\mathrm{a}+(\mathrm{N}+\mathrm{M})-(v+\mu)}=\mathbb{N}_{\mathrm{a}+1} \subseteq \mathcal{D}\left\{\Delta^{\mathrm{P}} \mathrm{f}\right\}
$$


Applying composition rule (3.9) from Theorem 3.4, we have for $\mathrm{t} \in \mathbb{N}_{\mathrm{a}+1}$,

$$
\begin{aligned}
& \Delta_{a+M-\mu}^{v} \Delta_{a}^{\mu} f(t) \\
& =\Delta_{a}^{P} f(t)-\sum_{j=0}^{M-1} \frac{\Delta_{a}^{j-M+\mu} f(a+M-\mu)}{\Gamma(-v-M+j+1)}(t-a-M+\mu) \frac{-v-M+j}{} .
\end{aligned}
$$

Observe that

$$
\lim _{\substack{v \rightarrow N^{-} \\ \mu \rightarrow(M-1)^{+}}}(t-a-M+\mu) \frac{-v-M+j}{}=(t-a-1) \frac{j-P-1}{L} \in(0, \infty)
$$

and

It follows that

$$
\lim _{v \rightarrow N^{-}} \frac{1}{\Gamma(-v-M+j+1)}=0
$$

$$
\lim _{\substack{v \rightarrow N^{-} \\ \mu \rightarrow(M-1)^{+}}}\left(\Delta_{a}^{P} f(t)-\Delta_{a+M-\mu}^{v} \Delta_{a}^{\mu} f(t)\right)=0,
$$

as expected (Compare this result to (3.6) in Lemma 3.2).

We are also interested in how these two operators compare for large t:

$$
\begin{aligned}
& \lim _{t \rightarrow \infty}\left(\Delta_{a}^{P} f(t)-\Delta_{a+M-\mu}^{v} \Delta_{a}^{\mu} f(t)\right)
\end{aligned}
$$

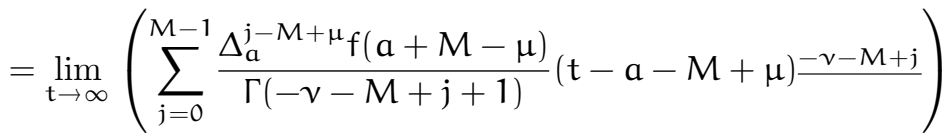

$$
\begin{aligned}
& =\sum_{j=0}^{M-1} \frac{\Delta_{a}^{j-M+\mu} f(a+M-\mu)}{\Gamma(-v-M+j+1)} \lim _{t \rightarrow \infty} \frac{\Gamma(t-a-M+\mu+1)}{\Gamma(t-a+P+1-j)} \\
& =0 \text {, }
\end{aligned}
$$

by applying the Squeeze Theorem, since $\mathrm{t}-\mathrm{a}-\mathrm{M}+\mu+1, \mathrm{t}-\mathrm{a}+\mathrm{P}+1-\mathrm{j} \geq 2$ for $\mathrm{t} \in \mathbb{N}_{\mathrm{a}+2}$ implies that

$$
0<\frac{\Gamma(t-a-M+\mu+1)}{\Gamma(t-a+P+1-j)} \leq \frac{\Gamma(t-a+1)}{\Gamma(t-a+2)}=\frac{1}{t-a+1} \stackrel{t \rightarrow \infty}{\rightarrow} 0 .
$$

We learn here that the discrepancy between a $\mathrm{P}^{\mathrm{th}}$-order difference and two composed fractionalorder differences who sum to $\mathrm{P}$ depends explicitly on how far $\mathrm{t}$ is away from the first point in their common domain, $\mathrm{a}+1$. Furthermore, we see this discrepancy vanish as $\mathrm{t}$ grows large.

The following table shows the first seven of these discrepancies in the specific case $\mathrm{f}(\mathrm{t})=\mathrm{e}^{\mathrm{t}}$, $a=0$ and $v=\mu=\frac{1}{2}$ :

\begin{tabular}{|c|c|c|c|c|c|c|c|}
\hline $\mathrm{t}$ & 1 & 2 & 3 & 4 & 5 & 6 & 7 \\
\hline$\Delta_{\frac{1}{2}}^{\frac{1}{2}} \Delta_{0}^{\frac{1}{2}} e^{\mathrm{t}}-\Delta e^{\mathrm{t}}$ & $\frac{1}{8}$ & $\frac{1}{16}$ & $\frac{5}{128}$ & $\frac{7}{256}$ & $\frac{21}{1,024}$ & $\frac{33}{2,048}$ & $\frac{429}{32,768}$ \\
\hline
\end{tabular}

Received: August 2010. Revised: September 2010. 


\section{References}

[1] FM Atici and PW Eloe, A Transform Method in Discrete Fractional Calculus, International Journal of Difference Equations, 22007 165-176.

[2] FM Atici and PW Eloe, Initial Value Problems in Discrete Fractional Calculus, Proc. Amer. Math. Soc., 1372009 981-989.

[3] FM Atici and PW Eloe, Discrete Fractional Calculus with the Nabla Operator, Electronic Journal of Qualitative Theory of Differential Equations, Spec. Ed. I 2009 1-12.B Kuttner, On Differences of Fractional Order, Proc. London Math. Soc., 71957 453-466.

[4] JB Diaz and TJ Olser, Differences of Fractional Order, Mathematics of Computation, 281974.

[5] Henry L Gray and N Zhang, On a New Definition of the Fractional Difference, Mathematics of Computation, 501988 513-529.

[6] W Kelley and A Peterson, Difference Equations: An Introduction with Application, Second Edition, Academic Press, New York, New York, 2000.

[7] KS Miller and B Ross, Fractional Difference Calculus, Proceedings of the International Symposium on Univalent Functions, Fractional Calculus and their Applications, Nihon University, Koriyama, Japan, 1988 139-152.

[8] K Oldham and J Spanier, The Fractional Calculus: Theory and Applications of Differentiation and Integration to Arbitrary Order, Dover Publications, Inc., Mineola, New York, 2002.

[9] I Podlubny, Fractional Differential Equations, Academic Press, New York, New York, 1999. 